Draft VERSION NOVEMBER 15, 2017

Preprint typeset using LATEX style AASTeX6 v. 1.0

\title{
THE TIME-DOMAIN SPECTROSCOPIC SURVEY: TARGET SELECTION FOR REPEAT SPECTROSCOPY
}

\author{
Chelsea L. Macleod ${ }^{1}$, Paul J. Green ${ }^{1}$, Scott F. Anderson ${ }^{2}$, Michael Eracleous ${ }^{3,4}$, John J. Ruan ${ }^{2}$, Jessie \\ Runnoe $^{5}$, William Nielsen Brandt ${ }^{3,4,6}$, Carles Badenes ${ }^{7}$, Jenny Greene ${ }^{8}$, Eric Morganson ${ }^{9,10}$, Sarah J. \\ Schmid $^{11}$, Axel Schwope ${ }^{11}$, Yue Shen ${ }^{10,9,12}$, Rachael Amaro ${ }^{1,10}$, Amy Lebled ${ }^{1,13}$, Nurten Filiz AK ${ }^{14}$, Catherine J. \\ Grier $^{3,4}$, Daniel Hoover ${ }^{2}$, Sean M. McGraw ${ }^{3,4}$, Kyle Dawson ${ }^{15}$, Patrick B. Hall ${ }^{16}$, Suzanne L. Hawley ${ }^{2}$, Vivek \\ Mariappan $^{15}$, Adam D. Myers ${ }^{17}$, Isabelle PÂris ${ }^{18}$, Donald P. Schneider ${ }^{3,4}$, Keivan G. Stassun ${ }^{19,20}$, Matthew A. \\ Bershady $^{21}$, Michael R. Blanton ${ }^{22}$, Hee-Jong SeO ${ }^{23}$, Jeremy Tinker ${ }^{22}$, J. G. Fernández-Trincado ${ }^{24,25}$, Kenneth \\ Chambers $^{26}$, Nick Kaiser ${ }^{26}$, R.-P. Kudritzki ${ }^{26}$, Eugene Magnier ${ }^{26}$, Nigel Metcalfe ${ }^{27}$, and Chris Z. Waters ${ }^{26}$ \\ ${ }^{1}$ Harvard Smithsonian Center for Astrophysics, 60 Garden St, Cambridge, MA 02138, USA \\ ${ }^{2}$ Department of Astronomy, University of Washington, Box 351580, Seattle, WA 98195, USA \\ ${ }^{3}$ Department of Astronomy \& Astrophysics, 525 Davey Laboratory, The Pennsylvania State University, University Park, PA 16802, USA \\ ${ }^{4}$ Institute for Gravitation and the Cosmos, The Pennsylvania State University, University Park, PA 16802, USA \\ ${ }^{5}$ Department of Astronomy, University of Michigan, 1085 S. University Avenue, Ann Arbor, MI 48109, USA \\ ${ }^{6}$ Department of Physics, The Pennsylvania State University, University Park, PA 16802, USA \\ ${ }^{7}$ Department of Physics and Astronomy and Pittsburgh Particle Physics, Astrophysics and Cosmology Center (PITT PACC), University of \\ Pittsburgh, 3941 O'Hara St, Pittsburgh, PA 15260, USA \\ ${ }^{8}$ Department of Astrophysical Sciences, Princeton University, Princeton, NJ 08544, USA \\ ${ }^{9}$ National Center for Supercomputing Applications, University of Illinois at Urbana-Champaign, 1205 W. Clark St., Urbana, IL 61801, USA \\ ${ }^{10}$ Department of Astronomy, University of Illinois at Urbana-Champaign, 1002 W. Green Street, Urbana, IL 61801, USA \\ ${ }^{11}$ Leibniz-Institut für Astrophysik (AIP), An der Sternwarte 16, 14482, Potsdam, Germany \\ ${ }^{12}$ Alfred P. Sloan Research Fellow \\ 13 Department of Physics \& Astronomy, Louisiana State Univiersity, 202 Nicholson Hall, Baton Rouge, LA 70803, USA \\ ' ${ }^{14}$ Faculty of Sciences, Department of Astronomy and Space Sciences, and Astronomy and Space Sciences Observatory and Research Center, \\ Erciyes University, 38039 Kayseri, Turkey \\ ${ }^{15}$ Department of Physics \& Astronomy, University of Utah, Salt Lake City, UT 84112, USA \\ ${ }^{16}$ Department of Physics \& Astronomy, York University, 4700 Keele Street, Toronto, Ontario M3J 1P3, CAN \\ ${ }^{17}$ Department of Physics and Astronomy, University of Wyoming, Laramie, WY 82071, USA \\ ${ }^{18}$ INAF - Osservatorio Astronomico di Trieste, Via G. B. Tiepolo 11, I-34131 Trieste, Italy \\ ${ }^{19}$ Vanderbilt University, Department of Physics \& Astronomy, 6301 Stevenson Center Ln., Nashville, TN 37235, USA \\ ${ }^{120}$ Fisk University, Department of Physics, 1000 17th Ave. N.,Nashville, TN 37235, USA \\ ${ }^{21}$ Department of Astronomy, University of Wisconsin-Madison, 475 N. Charter St., Madison, WI 53706, USA \\ ${ }^{22}$ Center for Cosmology and Particle Physics, Department of Physics, New York University, 4 Washington Place, New York, NY 10003, USA \\ ${ }^{23}$ Department of Physics and Astronomy, Ohio University, 251B Clippinger Labs, Athens, OH 45701, USA \\ ${ }^{24}$ Departamento de Astronomía, Universidad de Concepción, Casilla 160-C, Concepción, Chile \\ ' ${ }^{25}$ Institut Utinam, CNRS UMR6213, Univ. Bourgogne Franche-Comté, OSU THETA, Observatoire de Besançon, BP 1615 , 25010 Besançon \\ Cedex, France \\ ${ }^{26}$ Institute for Astronomy, University of Hawaii at Manoa, Honolulu, HI 96822, USA \\ ${ }^{27}$ Department of Physics, University of Durham Science Laboratories, South Road Durham DH1 3LE, UK
}

\section{ABSTRACT}

As astronomers increasingly exploit the information available in the time domain, spectroscopic variability in particular opens broad new channels of investigation. Here we describe the selection algorithms for all targets intended for repeat spectroscopy in the Time Domain Spectroscopic Survey (TDSS), part of the extended Baryon Oscillation Spectroscopic Survey within the Sloan Digital Sky Survey-IV. Also discussed are the scientific rationale and technical constraints leading to these target selections. The TDSS includes a large "Repeat Quasar Spectroscopy" (RQS) program delivering $\sim 13,000$ repeat spectra of confirmed SDSS quasars, and several smaller "Few-Epoch Spectroscopy" (FES) programs targeting specific classes of quasars as well as stars. The RQS program aims to provide a large and diverse quasar data set for studying variations in quasar spectra on timescales 
of years, a comparison sample for the FES quasar programs, and opportunity for discovering rare, serendipitous events. The FES programs cover a wide variety of phenomena in both quasars and stars. Quasar FES programs target broad absorption line quasars, high signal-to-noise ratio normal broad line quasars, quasars with double-peaked or very asymmetric broad emission line profiles, binary supermassive black hole candidates, and the most photometrically variable quasars. Strongly variable stars are also targeted for repeat spectroscopy, encompassing many types of eclipsing binary systems, and classical pulsators like RR Lyrae. Other stellar FES programs allow spectroscopic variability studies of active ultracool dwarf stars, dwarf carbon stars, and white dwarf/M dwarf spectroscopic binaries. We present example TDSS spectra and describe anticipated sample sizes and results.

Keywords: quasars: general - surveys - stars: variables: general

\section{INTRODUCTION}

With the massive photometric datasets expected from the next generation of time-domain imaging surveys, classification algorithms will become increasingly dependent on our understanding of cosmic variables. Recently, the Sloan Digital Sky Survey (SDSS)-IV (Blanton et al. 2017) extended Baryon Acoustic Oscillation Sky Survey (eBOSS; Dawson et al. 2016) has enabled spectroscopy of celestial variables through the Time Domain Spectroscopic Survey (TDSS). TDSS has been operational since August 2014 and had obtained 47,000 spectra of stars and quasars as of July 2016. The target selection for the main TDSS single-epoch-spectroscopy (SES) program, in which optical point sources (unconfirmed quasars and stars) are targeted based on variability for a first epoch of spectroscopy, prioritized to achieve a typical surface density on the sky of $\sim 10 \mathrm{deg}^{-2}$, is described in Morganson et al. (2015). Initial results from a pilot SES survey during SDSS-III (Eisenstein et al. 2011) are presented in Ruan et al. (2016a).

Aside from the discovery and classification of the variable sky, the spectroscopic variability of some classes of objects is of considerable interest. For example, time-domain spectroscopy of broad absorption line quasars was included in SDSSIII (e.g., Filiz Ak et al. 2012, 2013, 2014). Rather than be satisfied with extant SDSS spectroscopy for heterogeneously targeted objects, TDSS intentionally seeks repeat spectroscopic observations for subsets of known stars and quasars that are interesting astrophysically via several "few-epoch spectroscopy" (FES) subprograms. Keeping within the tight overall TDSS total fiber-budget of $\sim 10 \mathrm{deg}^{-2}$, each distinct FES subclass is approximately aimed to include of order $10^{2-3}$ objects per FES subclass, i.e., the minimum needed for various reasons to achieve better than $\sim 10 \%$ statistics per subclass. With the actual FES subclasses implemented, this then means that of order $\sim 10 \%$ of the total TDSS fiber-budget is allotted to FES, yielding an average target density of $1 \mathrm{deg}^{-2}$.

Recently, an eBOSS emission line galaxy (ELG) survey spanning 300 plates began observations in the Fall of 2016 (Raichoor et al. 2017), covering some areas of the sky previously observed in SDSS-IV. For the TDSS fiber allotment of $\sim 10 \mathrm{deg}^{-2}$ on these plates, we considered three main options for a targeting strategy: $(i)$ probing deeper into the photometrically variable density, confirming new SES variables with enhanced completeness but reduced purity, (ii) re-observing previous SES targets, therefore obtaining repeat spectroscopy for variable quasars and stars in general, or (iii) shifting to a greater quasar emphasis, targeting more quasars previously observed in those fields. Since the ELG survey overlaps existing SDSS-IV fields as well as regions not yet covered by SDSS-IV, we adopted a new target selection for these plates, and chose to acquire repeat spectra of quasars already known in the field (option iii); this choice also serves as a pilot for a potentially larger program in future all-sky spectroscopic surveys. In this paper, we describe all the non-SES TDSS sub-programs that select objects for multiple spectroscopic observations, including several smaller FES programs covering the full SDSS sky area, and this pilot program of repeat quasar spectroscopy (RQS) on the $\left(\approx 1200 \mathrm{deg}^{2}\right)$ area encompassed by the ELG plates.

Astrophysically, the main contributors to the variable sky, and thus the TDSS repeat spectroscopy sub-programs, are quasars and variable stars. The hallmarks of quasar spectra include the power-law continuum from a thermally emitting accretion disk, broad emission lines (BELs) from the broad line region (BLR) that are photoionized by a higher-energy UV continuum (Peterson 1993), and narrow emission lines (see review by Osterbrock \& Mathews 1986). The Balmer lines $(\mathrm{H} \alpha, \mathrm{H} \beta$, etc.) have historically been extremely useful for inferring information about the physical structure and dynamics of the BLR, and are directly related to the number of ionizing photons from the continuum source (Korista \& Goad 2004). In rare cases, these lines can be double peaked when feeble winds allow a low optical depth sightline to the outermost part of the BLR disk (Eracleous et al. 2009). The Balmer lines also form the basis 
of our "Type I" vs. "Type II" classification scheme, and repeat spectroscopy has been useful in the past to identify contaminants (e.g., with weak, broad Balmer emission lines) in samples of Type II quasars (Barth et al. 2014). The formation of other BELs is thought to involve more complicated processes (Waters et al. 2016). For example, the Mg II BEL is subject to collisional de-excitation and can show different emission properties than the Balmer lines (Roig et al. 2014; Cackett et al. 2015; Sun et al. 2015). The UV lines (e.g., C IV, N V) are known to trace winds (Richards et al. 2011). Broad absorption lines (BALs), found in 10-25\% of quasars (e.g., Weymann et al. 1991; Trump et al. 2006; Gibson et al. 2009), are believed to be formed in a wind that is launched from the accretion disk at 10-100 light days from the supermassive black hole (e.g., Murray et al. 1995; Proga 2000). A larger fraction of quasar spectra show narrow absorption lines (NALs; e.g., Lundgren et al. 2009), and some have absorption lines of intermediate widths (mini-BALs). ${ }^{1}$ The existence of BAL variability that has been systematically studied since SDSS-III (Filiz Ak et al. 2012, 2013, 2014) has been known for almost three decades (see review by Turnshek 1988).

The menagerie of stellar variable classes encompasses causes both intrinsic (e.g., pulsators like RR Lyrae, Cepheids, and long-period variables and eruptive stars like CVs, novae, and symbiotics) and observer-dependent (e.g., eclipsing and/or spectroscopic binaries and rotation) variables. Photometrically, a few percent of all stars are considered variable, with the exact number depending on the filter bands used, the cadence of observations, and the limiting magnitude of the sample. With dense photometric monitoring, a few percent of those will show periodic variability (arising from radial pulsations, rotation or orbital motion). The remaining variable stars exhibit eruptive or irregular variability, the latter class including flaring stars across the main sequence (e.g., Davenport 2016). The periodic stellar variables can be classified with reasonable efficiency based on their period, amplitude, and lightcurve shape (e.g., Palaversa et al. 2013; Drake et al. 2014; VanderPlas \& Ivezić 2015). Spectroscopy provides further important physical characteristics such as gravity, temperature, and radial velocity. However, single-epoch spectroscopy captures but a single phase, and may provide little insight into the physical reason for the observed variability. Multi-epoch spectra, in contrast, can provide key information about e.g., radial velocity variations and orbital properties of binaries, or emission line variability related to chromospheric activity, irradiation, or accretion. Spectroscopic variability surveys in broad stellar samples are virtually nonexistant to date, but can broadly characterize stellar variability in physical detail, and also focus on specific classes of stars that are known or suspected to be variable.

We describe the TDSS input data sets used for choosing our spectroscopic targets in $\S 2$. In the following sections, we present the selection algorithms used by various subprograms of TDSS to target objects for repeat spectroscopy, with the FES programs described in $\S 3$ and the RQS program in $\S 4$. We summarize these programs in $\S 5$.

\section{INPUT DATA SETS}

Spectra from the first two phases of the SDSS (York et al. 2000, also referred to as SDSS-I/II) form the basis of most samples targeted here. The SDSS legacy survey includes observations up through Data Release (DR) 7 (Abazajian et al. 2009). The SDSS-III survey continued to extend the imaging and spectroscopic sky coverage of the SDSS surveys, culminating with DR12 (Alam et al. 2015). The SDSS-IV project eBOSS began in July 2014, marking the formal start of TDSS observations. The most recent data release is DR13 (SDSS Collaboration et al. 2016).

We use imaging data from the SDSS-I/II/III and Pan-STARRS-1 (PS1; Kaiser et al. 2002) $3 \pi$ surveys. SDSS started its imaging campaign in 2000 and concluded in 2007, having covered 11,663 $\mathrm{deg}^{2}$. SDSS-III added $\sim 3000 \mathrm{deg}^{2}$ of new imaging area in 2008. PS1 imaging commenced in 2009 and provided light curves for all SDSS sources through 2013. Hence, the addition of the PS1 photometry to the SDSS photometry increases the span of light curves from $\approx 8$ to $\approx 14$ years. More details on each survey and the photometric variability measures used in (§3.4) and ( $(4)$ are described in this section.

\subsection{SDSS Imaging}

The SDSS uses the imaging data gathered by a dedicated $2.5 \mathrm{~m}$ wide-field telescope (Gunn et al. 2006), which collected light from a camera with $302 \mathrm{k} \times 2 \mathrm{k}$ CCDs (Gunn et al. 1998) over five broad bands - ugriz (Fukugita et al. 1996; Doi et al. 2010) - in order to image 14,555 unique $\operatorname{deg}^{2}$ of the sky. This area includes $7,500 \mathrm{deg}^{2}$ in the North Galactic Cap (NGC) and 3,100 $\mathrm{deg}^{2}$ in the South Galactic Cap (SGC). The Eighth Data Release (DR8; Aihara et al. 2011) provides the full imaging data set and updated photometric calibrations. This catalog provides the magnitudes and astrometry used in constructing our target samples; all coordinates hereafter are J2000.

The Stripe 82 region of SDSS (S82; $-60^{\circ}<\alpha<50^{\circ}$ and $|\delta|<1.27^{\circ}$ ) covers $\sim 300 \mathrm{deg}^{2}$ and has been observed $\sim 60$ times on average to search for transient and variable objects (Frieman et al. 2008; Abazajian et al. 2009). These multi-

${ }^{1}$ Operationally, BALs have widths $>2000 \mathrm{~km} / \mathrm{s}$, NALs have widths $<500 \mathrm{~km} / \mathrm{s}$, and mini-BALs are in-between. 
epoch data probe time scales ranging from 3 hours to 8 years and provide well-sampled 5-band light curves. The S82 variable and standard star catalogs (Ivezić et al. 2007) are used to train the TDSS SES selection in Morganson et al. (2015), and therefore the hypervariables selection (§3.4). The S82 data are used for variability selection in the RQS program $(\S 4)$.

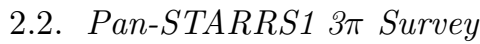

PS1 utilizes a $1.8 \mathrm{~m}$ telescope equipped with a 1.4-gigapixel camera. Over the course of 3.5 years of the $3 \pi$ survey, up to four exposures per year in 5 bands, $g_{\mathrm{PS} 1}, r_{\mathrm{PS} 1}, i_{\mathrm{PS} 1}, z_{\mathrm{PS} 1}, y_{\mathrm{PS} 1}$ have been taken across the entire $\delta>-30^{\circ}$ sky (for full details, see Tonry et al. 2012; Metcalfe et al. 2013). Each nightly observation consists of a pair of exposures separated by $15 \mathrm{~min}$ to search for moving objects. For each exposure, the PS1 $3 \pi$ survey has a typical $5 \sigma$ depth of 22.0 in the $g$-band (Inserra et al. 2013). The instrumentation, photometric system, and the PS1 surveys are described in Kaiser et al. (2010), Stubbs et al. (2010), and Magnier et al. (2013), respectively. A high quality subset of PS1 data was first released in Processing Version (PV) 1, and PV2 added data through a later date in the observing, as well as some previously missed earlier observations via better analysis failure handling (for an overall description of the PS1 database, see Flewelling et al. 2016).

\subsection{Variability Measures}

Whereas variability selection formed the basis for the first epoch (SES) target selection described in Morganson et al. (2015), multiepoch imaging data were also used to select known stars and quasars for the extremely variable (or "hypervariable"; §3.4) and RQS quasar (§4) samples. The SES selection uses a three-dimensional parameter space (magnitude, PS1-only variability, and SDSS-PS1 difference), designed to achieve a high-purity variable sample at a typical surface density on the sky of $\sim 10 \mathrm{deg}^{-2}$. As part of the SES selection, hypervariables were selected based on a single variability metric $V$ that parameterizes variability in a two-dimensional space of two variability terms $S_{1}$ and $S_{2}$ :

$$
V=\left(\operatorname{median}\left(\left|\operatorname{mag}_{\mathrm{PS} 1}-\operatorname{mag}_{\mathrm{SDSS}}\right|\right)^{2}+4 \operatorname{median}\left(\operatorname{Var}_{\mathrm{PS} 1}\right)^{2}\right)^{1 / 2}=\left(S_{1}^{2}+4 S_{2}^{2}\right)^{1 / 2},
$$

(Equation 11 of Morganson et al. 2015). Qualitatively, $S_{1}$ is the PS1-SDSS difference and represents long term (multiyear) variability, and $S_{2}$ is the PS1-only variability characterizing short term (days to a few years) variability. This non-standard variability measure was intended to combine short term variability and long term variability measures into one quantity. The training sets used to derive this quantity, as well as the detailed statistics of this selection can be found in Morganson et al. (2015). Hypervariables with $V>2$ mag were prioritized during the SES selection, whereas for the FES targets, a lower $V$ threshold is used (see §3.4). As for the SES selection, an updated version of the "ubercalibrated" PS1 data (Schlafly et al. 2012), which include PV1 data up through July 2013, was used to select FES hypervariables. For detailed definitions, see Morganson et al. (2015).

The RQS program targets known SDSS quasars based on median SDSS magnitude and highly significant variability (see $\S 4$ ). Here, the variability selection is based on the reduced $\chi^{2}$ of the light curve:

$$
\chi_{p d f}^{2}=\frac{1}{n-1} \sum_{i=1}^{n}\left(\frac{m_{i}-\mu}{\sigma_{i}}\right)^{2},
$$

where $n$ is the number of data points in a given filter, $m_{i}, \ldots, m_{n}$ are the individual magnitudes, $\sigma_{i}$ is the error associated with $m_{i}$, and $\mu$ is the mean magnitude. The reduced $\chi_{p d f}^{2}$ in both $g$ and $r$ bands is used to define the variability-selected subsamples (see $\S 4$ for detailed criteria). The $\chi_{p d f}^{2}$ cuts remove noisy, sparse light curves where the variability is at low signal-to-noise ratio (SNR; e.g., see Figure 1), rather than removing quasars that do not intrinsically vary, since essentially all quasars should be variable in the absence of poor photometry or systematics (Butler \& Bloom 2011).

For the $\chi_{p d f}^{2}$ calculation, all primary and secondary SDSS photometric observations are considered, along with PS1 PV2 data, matched to within $1^{\prime \prime}$ and without regard to morphology or data quality flags. The PS1 data include observations up through December 2013, and the error inflations derived in Morganson et al. (2015) are applied. Point source (PSF) magnitudes are adopted, as we are interested in the nuclear variability (e.g., in the case of resolved AGN). Before the $\chi_{p d f}^{2}$ calculation, the SDSS magnitudes are transformed to the PS1 system as described in Morganson et al. (2015). We also first remove deviant points, as defined by being $>0.5$ mag from either the SDSS or PS1 running average. For this outlier rejection, we consider SDSS and PS1 data separately due to the gap in time between the two data sets. The outlier rejection is only applied to SDSS light curves with $n \geq 10$ points (i.e., Stripe 82 data), and to 
PS1 data with $n \geq 5$ points. To compute the running averages, we use a window of five points for SDSS data and three points for PS1 data. The outlier rejection affects 5\% (10\%) of the PS1 (Stripe 82) light curves. Among these, $12.5 \%(7 \%)$ of the PS1 (Stripe 82 ) epochs on average are removed as a result. ${ }^{2}$

Figure 1 shows example light curves for four sources with S82 and PS1 photometry and with different values of $\chi_{p d f}^{2}$.

\subsection{Spectroscopic Data}

All samples are constructed based on known spectroscopic classifications in SDSS. The BOSS spectrographs and their SDSS predecessors are described in detail by Smee et al. (2013). SDSS-III BOSS (Dawson et al. 2013) significantly expanded the coverage in the SGC (approximately $-2^{\circ}<\delta<35^{\circ},-30^{\circ}<\alpha<30^{\circ}$, see Figure 2), and revisited the entire NGC area. Since the FES targets were planned before the start of SDSS-IV, they were restricted to SDSSI/II/III observations. SDSS-IV eBOSS observations are planned to cover the entire SGC BOSS footprint and about half the NGC, targeting mostly quasars and galaxies (Blanton et al. 2017). Since the RQS targets were compiled in summer 2016, they also draw from newly confirmed quasars targeted as part of SDSS-IV (Myers et al. 2015;

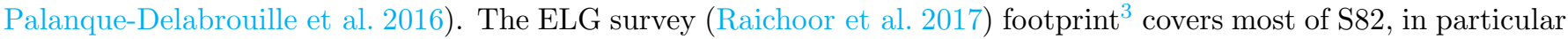
the "Thin82" and "Thick82" regions outlined in Figure 2, totalling $620 \mathrm{deg}^{2}$ in the SGC. This area is also covered by eBOSS plates designed for luminous red galaxies (LRG; Prakash et al. 2016) and quasar targets (including some that were observed after the SDSS-IV observations shown in Figure 2). The ELG footprint also includes $600 \operatorname{deg}^{2}$ in the NGC.

The wavelength coverage of the SDSS (BOSS) spectrographs is $3800-9200 \AA(3600-10,400 \AA)$, with a spectral resolution ranging from 1850 to 2200 (1560 to 2650). The SDSS and TDSS spectra presented in this work all have $\lambda_{\text {eff }}=5400 \AA$, i.e., the plate holes were drilled to maximize the SNR at $\lambda_{\text {eff }}$, and the BOSS spectra either have $\lambda_{\text {eff }}=5400 \AA$ or $\lambda_{\text {eff }}=4000 \AA$ (Dawson et al. 2013). To accurately compare spectra with differing $\lambda_{\text {eff }}$, one must correct the spectra using the prescriptions given in Margala et al. (2016); Guo \& Gu (2016); Harris et al. (2016). Note that the Margala et al. (2016) corrections are applied in the DR14 release of spectra from the BOSS spectrographs (Abolfathi et al. 2017).

\subsubsection{Quasar Catalogs and Temporal Baselines}

As described by Richards et al. (2002), the bulk of SDSS quasar target candidates in SDSS-I/II were selected for spectroscopic observations based on their optical colors and magnitudes in the SDSS imaging data or their detection in the FIRST radio survey (Becker et al. 1995). Low-redshift, $z \lesssim 3$, quasar targets were selected based on their location in ugri-color space and the quasar candidates passing the ugri-color selection were selected to a flux limit of $i=19.1$. High-redshift $(z \gtrsim 3)$ objects were selected in griz-color space and are targeted to $i=20.2$. Furthermore, if an unresolved, $i \leq 19.1$ SDSS object was matched to within $2^{\prime \prime}$ of a source in the FIRST catalog, it was included in the quasar selection. Additional quasars were also (inhomogeneously) discovered and cataloged in SDSS-I/II using $\mathrm{X}$-ray, radio, and/or alternate odd-color information, and extending to fiber-magnitudes of about $m<20.5$ (e.g., see Anderson et al. 2003).

Unless otherwise stated, we select quasars for repeat spectroscopy from one of the visually vetted quasar catalogs: the SDSS-I/II DR5/7 quasar catalogs (DR5Q, DR7Q; Schneider et al. 2007, 2010; Shen et al. 2011) or the DR12 quasar catalog (DR12Q, final quasar catalog of SDSS-III; Pâris et al. 2017). The RQS target selection considers confirmed SDSS-IV quasars from post-DR13 data (Myers et al. 2015; Palanque-Delabrouille et al. 2016), specifically the SpAll database version v5_9_1, which covers a region in the SGC (see Figure 2 and Table A1 for the SDSS-IV coverage at the time of target selection). The following SDSS-IV spectra are excluded from consideration:

1. Those objects lacking primary (mode $=1$ ) magnitudes in DR10 (these objects are faint and few in number);

2. Those with OBJTYPE $=$ SKY;

3. Those objects with morphological TYPE $=0$, according to DR10 photometry; and

4. Those at redshift $z \geq 0.8$ with morphological TYPE $=3$, since a resolved quasar should be at a lower redshift.

In order to determine the number of existing spectra in $\S 4$, we extract all spectroscopy within $2^{\prime \prime}$ from the SDSS DR12 SpecObjAll database. For the new SDSS-IV objects, we use the NSPEC field from SpAll-v5_9_1.

\footnotetext{
${ }^{2}$ While this criterion could lead to the rejection of some interesting variables, we are mainly interested in maximizing the sample efficiency by rejecting spurious data points that may otherwise lead to a misleading variability measure.

3 Note: the ELG footprint has been updated since the version shown in Figure 1 of Morganson et al. (2015).
} 


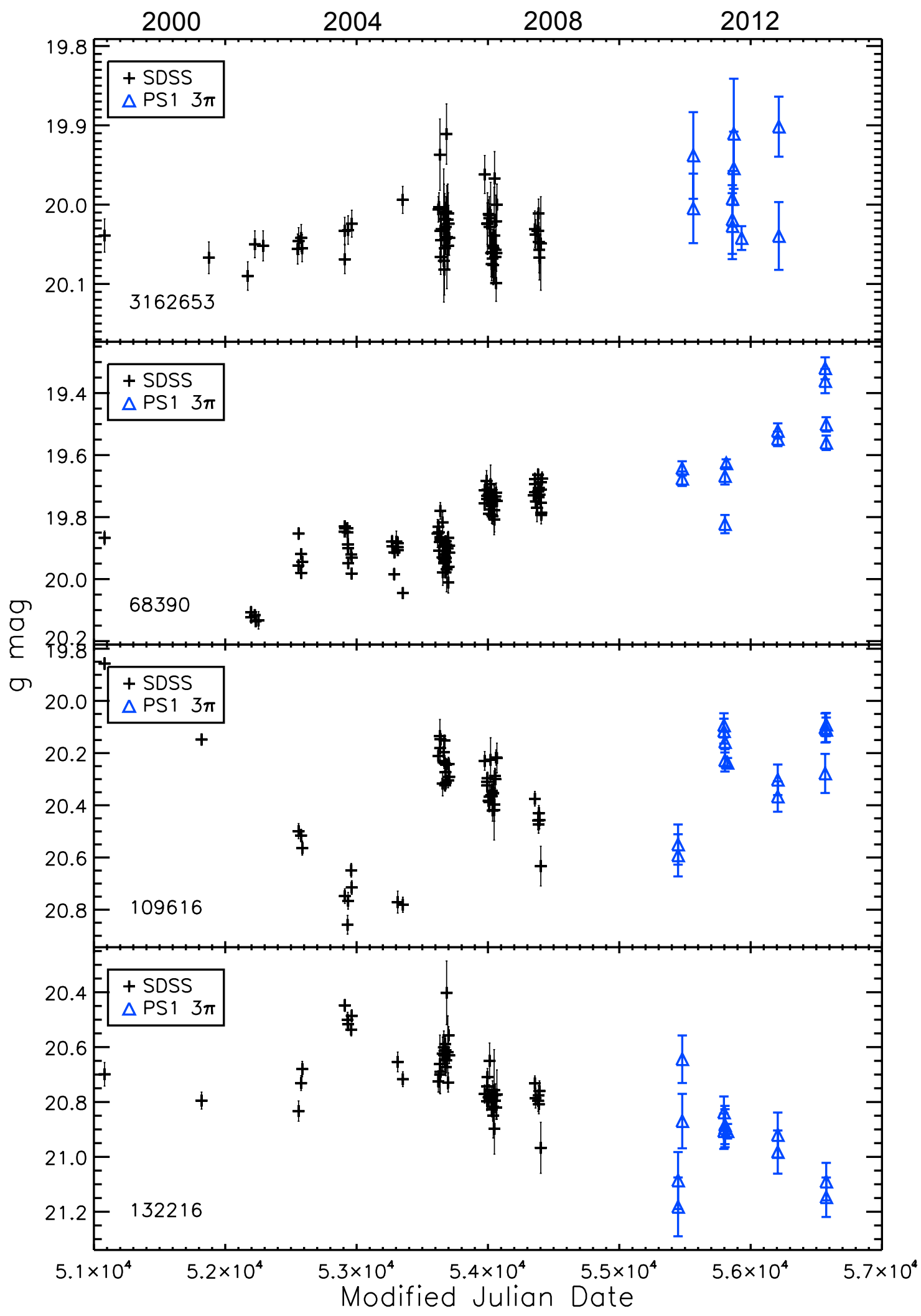

Figure 1. Example light curves of known quasars in Stripe 82. The first panel shows an example with relatively insignificant variability $\left(\chi_{p d f}^{2}<3\right)$. The quasars in the remaining panels show highly significant variability $\left(\chi_{p d f}^{2}>30\right)$ and are thus selected as RQS targets (see $\S 4$ ). 


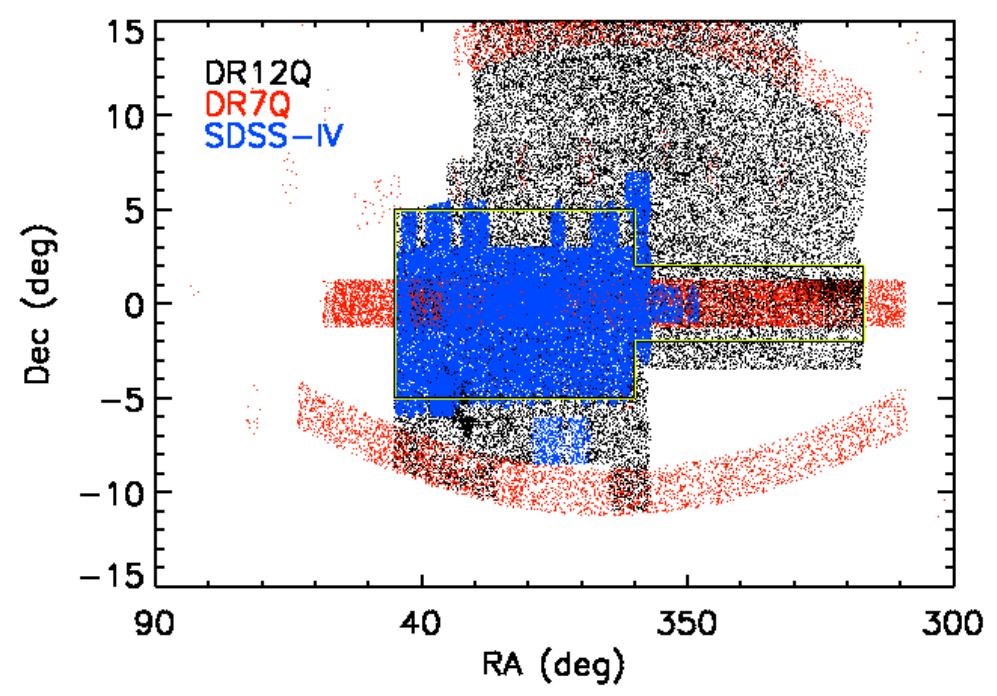

Figure 2. Sky coverage in the SGC (J2000 coordinates) as of early 2016 (SpAll version v5_9_1). The "Thin82" and "Thick82" chunks are outlined, with Thin 82 covering a subset of $315^{\circ}<\alpha<360^{\circ},-2.0^{\circ}<\delta<2.75^{\circ}$, and Thick 82 covering $0^{\circ}<\alpha<45^{\circ}$, $-5^{\circ}$ Figures $3^{\circ}$ and 4 show the anticipated distribution of time lags between spectra for SDSS quasars. Figure 4 also shows the existing distribution of time lags and displays these distributions as a function of absolute magnitude $M_{i}$, where the $M_{i}$ values are estimated from the apparent $i$ magnitudes and distance modulus (no K-correction is applied). Note that these Figures do not include the well-sampled quasar cadence from the SDSS Reverberation Mapping Program (Shen et al. 2015).

\section{FEW-EPOCH SPECTROSCOPY (FES)}

In addition to its main program to obtain initial characterization spectra of $>10^{5}$ optical variables selected from PS1, the TDSS includes nine separate smaller, FES programs to study spectroscopic variability. The FES programs target objects with existing SDSS spectroscopy amongst classes of quasars and stars of particular astrophysical interest to build statistical samples for follow-up study. ${ }^{4}$ These include, in approximate order of decreasing sample size: BAL quasars, the most photometrically variable ("hypervariable") quasars, high SNR normal broad-line quasars, quasars with double-peaked or very asymmetric broad emission line profiles, hypervariable stars (including the most highly variable classical pulsators), active ultracool (late-M and early-L) dwarf stars with $\mathrm{H} \alpha$ emission, dwarf carbon stars, white dwarf/M dwarf spectroscopic binaries with $\mathrm{H} \alpha$ emission, and binary supermassive black hole candidates from Mg II broad line velocity shift analysis.

The FES programs and respective scientific goals and target selections are described in the following sections, starting with stars (§3.1-3.4) and ending with quasars (§3.4-3.8). The target flags used are listed in each subsection heading.

\subsection{Magnetic activity on late- $M$ and early- $L$ dwarfs (TDSS_FES_ACTSTAR)}

Magnetic activity is ubiquitous in stars at the transition between the M and L spectral types (ML dwarfs). In optical spectra, this activity is best identified with the $\mathrm{H} \alpha$ emission line, which traces chromospheric heating on these low mass objects (e.g., Gizis et al. 2002; West et al. 2011). $\mathrm{H} \alpha$ is the optimal diagnostic in part because it is found in a relatively red portion of the spectrum, so is easier to observe for these very cool, red objects. Serendipitous and dedicated observations of $\mathrm{H} \alpha$ emission on multiple timescales have indicated $\mathrm{H} \alpha$ emission varies (sometimes dramatically; Hall 2002) on multiple timescales (e.g., Berger et al. 2009; Schmidt et al. 2015). Chromospheric heating covers only a small portion of the surface ( $<1 \%$ Schmidt et al. 2015), and those regions rotate in and out of view on timescales of hours to days (due to relatively rapid rotation; Reiners \& Basri 2008) leading to $\mathrm{H} \alpha$ variability. On timescales of weeks to months, we expect the chromospheric emission regions to change in size due to underlying shifts

\footnotetext{
${ }^{4}$ Note that the FES program approach is conceptually somewhat different than, and complementary to, the RQS approach. The RQS intentionally - and with fewer a priori biases - samples spectral variability across a much broader range of quasar subclasses, whereas the FES subclasses are more-specific-science focused (e.g., BALQSOs), and therefore efficiently address some more restricted questions. The FES subclasses are custom-tuned and relatively smaller than the RQS, but still large in sample-size in an absolute sense with hundreds to thousands each, providing excellent statistics albeit attuned to more highly- and specifically-selected subsamples.
} 


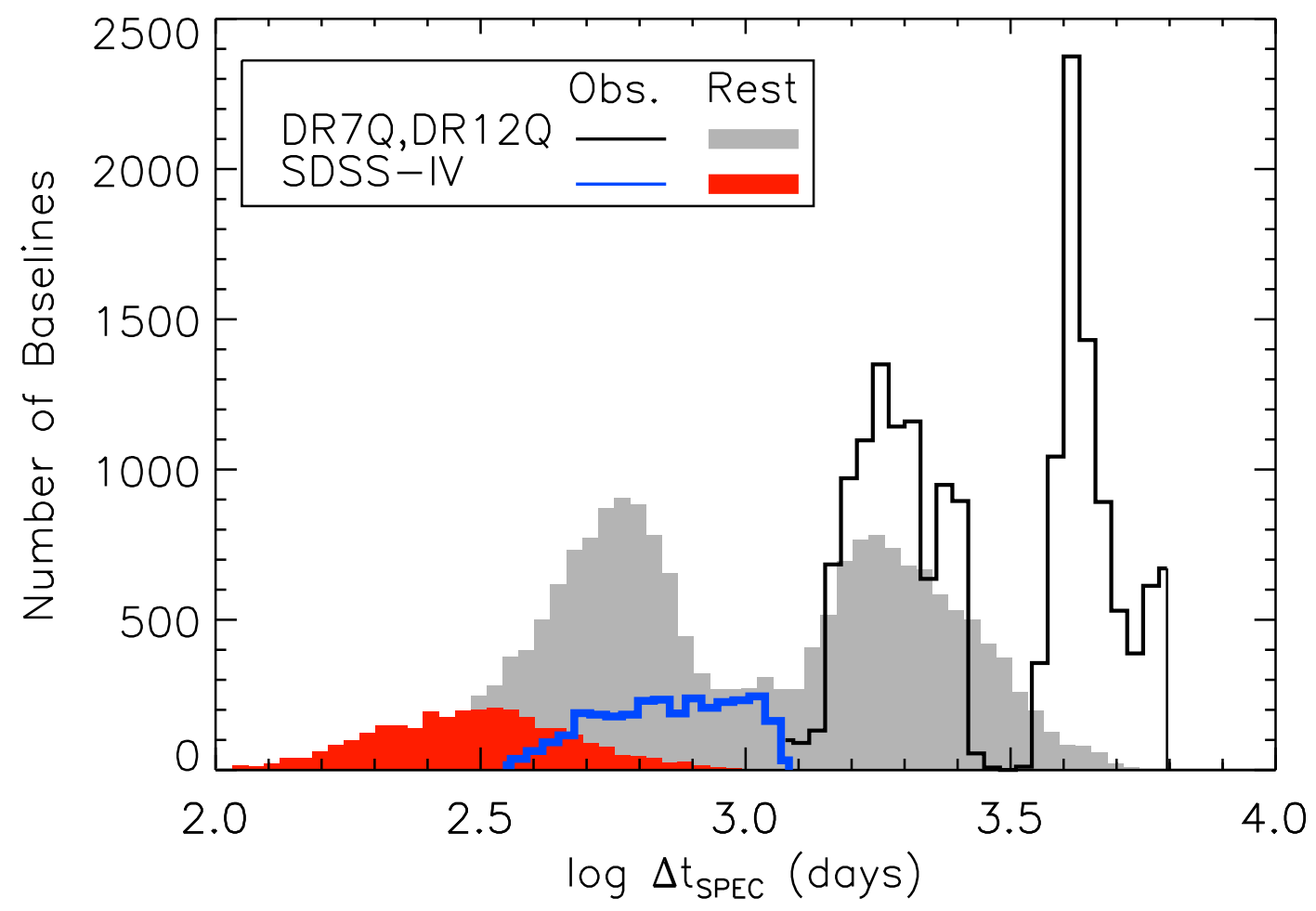

Figure 3. Distribution of anticipated time baselines in the observed and rest frames (open and filled histograms, respectively) for quasars considered for repeat spectroscopy in the SDSS-IV RQS program ( $₫ 4$ ). The RQS epochs are artificially set to a uniform distribution over the year 2017. The total distribution of $\sim 20,000$ quasar baselines in the observed (rest) frame is shown as the open black (filled gray) histogram, where a single existing spectroscopic epoch is adopted. The baselines in the observed (rest) frame for objects with existing SDSS-IV spectra ( 3,000 total) are shown as the open blue (filled red) histogram.

in the magnetic field (similar to shifts in sunspots). $\mathrm{H} \alpha$ may also show variability over year to decade-long timescales based on long-timescale magnetic field changes that are similar to the 11-year solar cycle. Analyses of $\mathrm{H} \alpha$ variability on ML dwarfs have so far been limited to $\sim 20$ objects serendipitously observed by multiple groups, but the data indicate that $30-50 \%$ of ML dwarfs exhibit significant variability over timescales that span months to years (Schmidt et al. 2015).

By comparing original spectra of ML dwarfs from the SDSS legacy survey with an additional spectrum from TDSS, we have a unique opportunity to monitor changes in $\mathrm{H} \alpha$ emission lines over timescales of $6-14$ years. These observations can either be taken as indicators of the level of overall variability, or could be combined with data over shorter timescales to detect decadal magnetic cycles. The SDSS data for ML dwarfs also allows three-dimensional Galactic kinematics that can be leveraged to examine age and activity correlations among the multi-epoch observations.

To select the FES sample of ML dwarfs, we combined the West et al. (2011) M dwarf and Schmidt et al. (2010) L dwarf catalogs. We selected a subset of dwarfs from those catalogs with magnitudes between $17<i<21$ and spectral types from M7 to L3. We also required an average SNR $>3$ per $1.5 \AA$ pixel in the continuum surrounding $\mathrm{H} \alpha(6530$ $6555 \AA$ and $6575-6600 \AA$ ) so that the presence and strength of $\mathrm{H} \alpha$ emission can be reliably measured (e.g., West et al. 2008). The dwarfs in our initial sample are contained in the BOSS Ultracool Dwarfs catalog (Schmidt et al. 2017, in prep.), and we required that each of the dwarfs have a photometric distance (based on SDSS photometry), proper motion (from SDSS-2MASS-WISE positions) and radial velocity (based on SDSS spectroscopy) from that catalog. We also restricted the sample to dwarfs within $300 \mathrm{pc}$ of the Galactic plane.

The selection criteria resulted in a total of $3739 \mathrm{M} 7,534 \mathrm{M} 8,153 \mathrm{M} 9$, and $23 \mathrm{~L}$ dwarfs. To reduce the sample to $\sim 1000$ ML dwarfs, we binned the data by height above the Galactic plane and restricted each 25 pc-wide bin to 60 dwarfs randomly drawn from each spectral type. The final target list includes 1036 stars (583 M7, 283 M8, 147 M9, and $23 \mathrm{~L}$ dwarfs). Initial data from the ML dwarf FES sample includes dwarfs that have no change in their activity level as well as those that have strong variability. The spectrum of a strongly active and variable M dwarf is shown in 


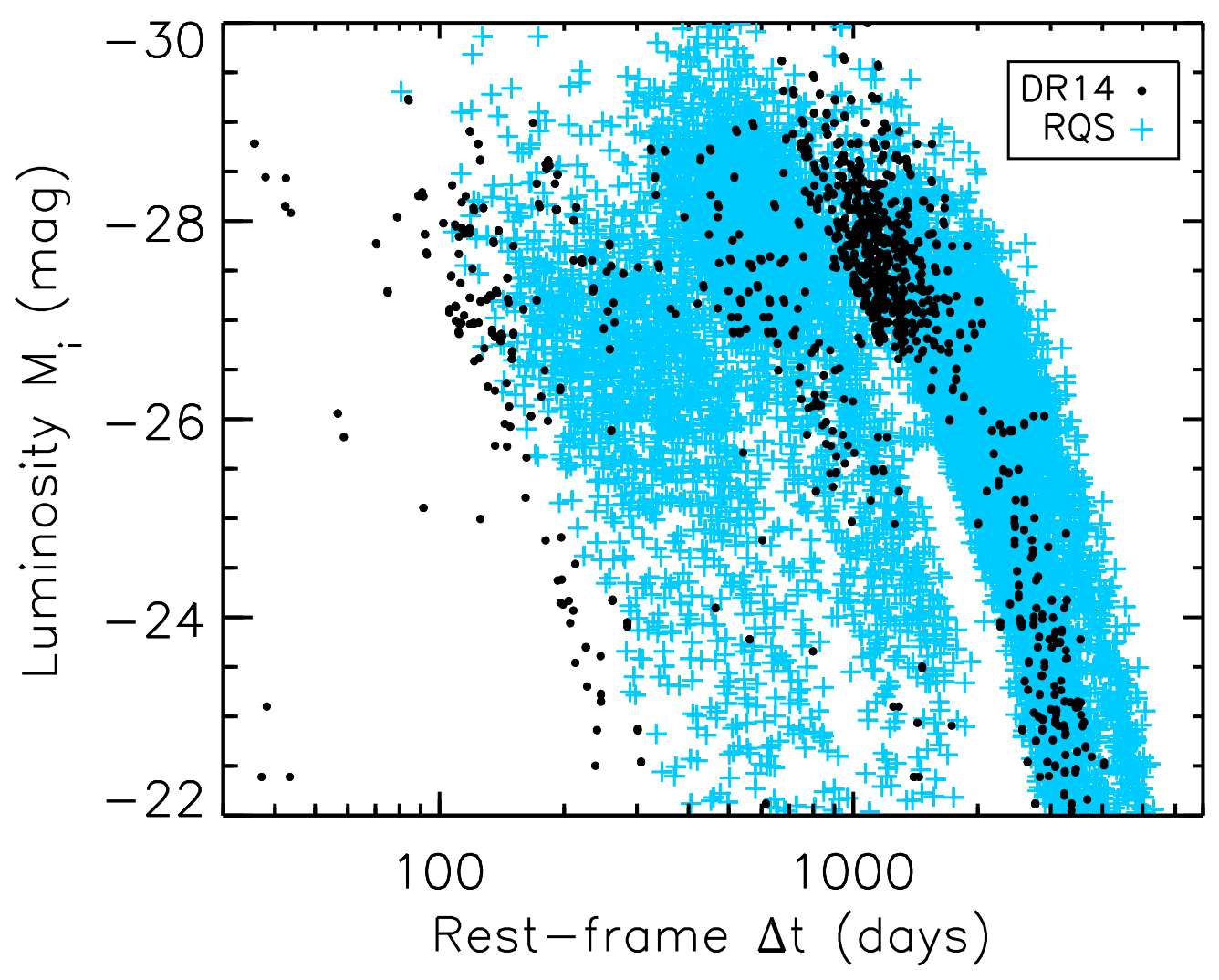

Figure 4. The distribution of rest-frame time intervals between spectra as a function of luminosity for $i<19$ quasars in DR14 is shown as black dots. The points are restricted to quasars within a representative area of $233 \mathrm{deg}^{2}$ that have more than one existing spectroscopic epoch, and include all existing pairs of epochs in DR14. The anticipated distribution for $i<19$ quasars targeted by the RQS program $(\S 4)$ is shown in cyan (adopting a single existing epoch for each quasar). The RQS epochs are artificially set to a uniform distribution over the year 2017.

Figure 5.

\subsection{White Dwarf-M Dwarf binaries (TDSS_FES_WDDM)}

Recent studies have demonstrated that close binaries consisting of white dwarf-M dwarf pairs (WD-dM binaries) have a significant effect on the magnetic activity of their main sequence components (e.g., Morgan et al. 2012). The binary separations where increased magnetic activity is observed extend to $\sim 100 \mathrm{AU}$. While many of the WD+dM are unresolved photometrically, the two components can be separated in low-resolution spectroscopy due to their vastly different spectral energy distributions. While there is evidence of increased magnetic activity in close pairs, there has been limited analysis of the variability of magnetic activity in binary systems. Variability studies can distinguish among possible causes of activity (e.g., irradiation, accretion, disk disruption, and spin-up). This program aims to re-observe $\sim 400 \mathrm{WD}$-dM binaries identified via their spectral energy distributions. By measuring the magnetic activity of the $\mathrm{M}$ dwarf via the $\mathrm{H} \alpha$ equivalent width (EW), the goals are to determine (i) the effect of binary separation on the variability of magnetic activity, (ii) the effect of rotation on stellar activity in close binaries, and (iii) the WD cooling age, spectral type, orbital parameters, metallicity, and Galactic height, and the corresponding effects on magnetic activity.

Starting with the WD-dM binary sample of Morgan et al. (2012), targets that met the following criteria were selected:

1. Within the magnitude range $17<i<21$,

2. Clear $\mathrm{H} \alpha$ emission from visual inspection, ${ }^{5}$ and

5 We found that simple EW and SNR criteria with fixed wavelength intervals led to unreliable results if there were significant shifts in 

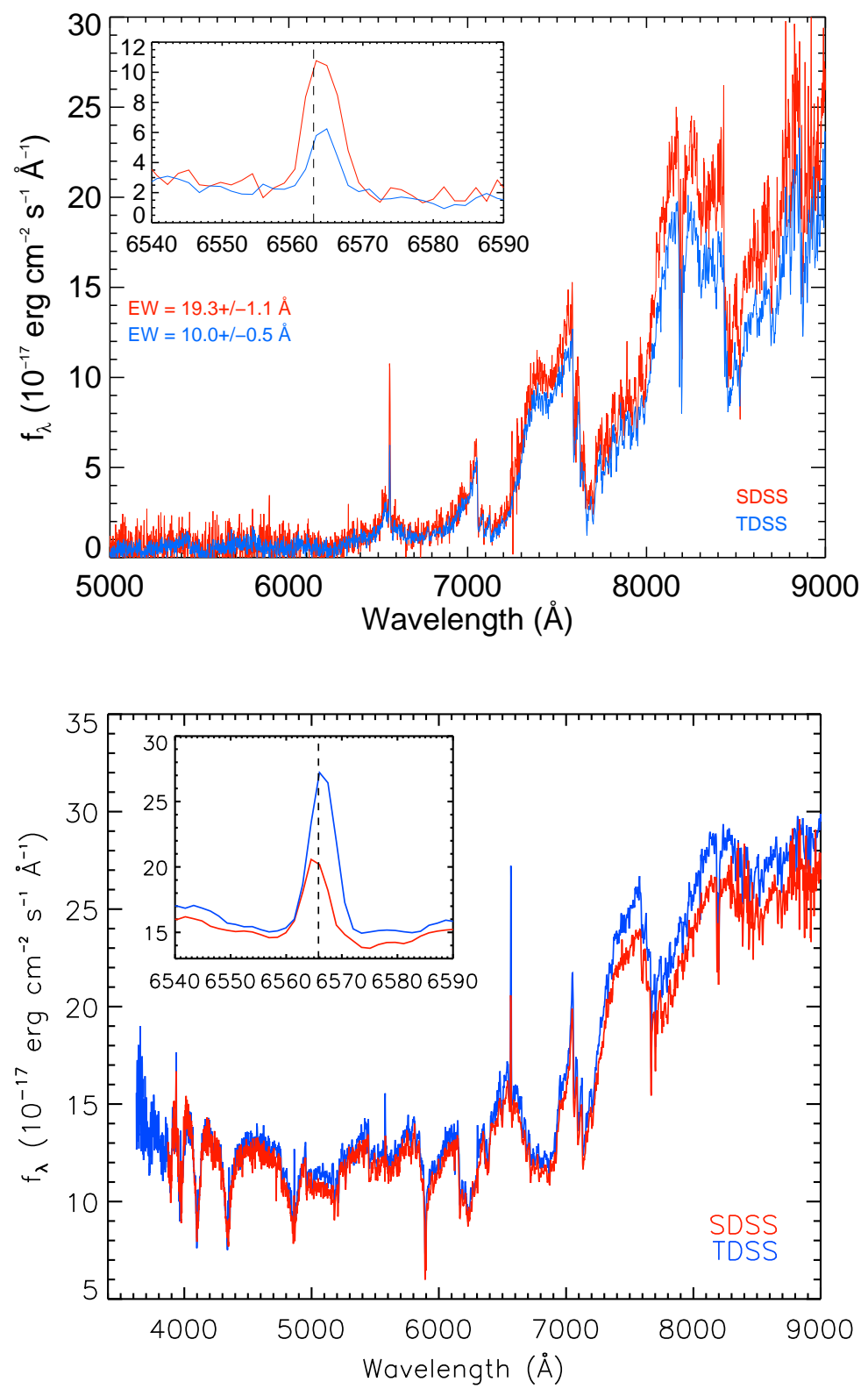

Figure 5. Top: SDSSJ 014724.37+005751.4, an M9 dwarf (target class TDSS_FES_ACTSTAR, see $\S 3.1$ ) first observed with the original SDSS spectrograph (black) with an additional epoch of spectroscopy from TDSS (red). The inset displays the area surrounding the $\mathrm{H} \alpha$ emission line. This object has strong variable $\mathrm{H} \alpha$ emission; between the original epoch (MJD 51793) and the more recent epoch (MJD 56902) the emission line weakened significantly. It is not yet known whether this behavior reflects variations on a short timescale or longer timescale evolution of the magnetic field. Bottom: SDSSJ 231105.67+220208.7, a WD-dM binary (target class TDSS_FES_WDDM, see §3.2) that shows a significant brightening of H $\alpha$ in the TDSS spectrum over $7.9 \mathrm{yr}$ (see inset). This object has a binary period of 13.9 hours (Nebot Gómez-Morán et al. 2011). The same level of smoothing has been applied to both SDSS and TDSS spectra, and the observed frame is shown.

3. Accurate proper motion measurements from the SDSS-USNOB proper motions table (MATCH=1; PMRA and PMDEC $\neq 0$; and DIST22 $>7^{\prime \prime}$, where DIST22 is the distance to the nearest neighbor with $g<22$ ). This criterion is necessary in order for the fiber holes to be drilled in the correct locations.

The resulting sample contains 402 active, WD-dM pairs that span several M dwarf spectral types. An example of a binary with variable $\mathrm{H} \alpha$ is shown in the bottom panel of Figure 5. 


\subsection{Variability in Dwarf Carbon Stars (TDSS_FES_DWARFC)}

Carbon in stellar atmospheres - indeed, most of the carbon in the universe - is produced by the triple- $\alpha$ process of helium fusion $\left(3^{4} \mathrm{He} \rightarrow{ }^{12} \mathrm{C}\right)$ in the interiors of red giant stars. Strong carbon molecular bands are historically expected to be seen only in asymptotic giant branch (AGB) stars that have experienced a third "dredge-up" (Iben \& Renzini 1983). However, among stars showing such $\mathrm{C}_{2}$ and $\mathrm{CN}$ molecular bands (C stars), the main sequence carbon dwarfs (dCs) are numerically dominant in the Galaxy (Green et al. 1992). The accepted explanation for dCs is that they must all be in post-mass transfer binaries, where the former AGB star has since become a white dwarf, leaving a carbonenhanced dC primary. Indeed, a handful of "smoking gun" systems reveal evidence for this evolutionary scenario, having composite spectra with a hot DA white dwarf component (Heber et al. 1993; Liebert et al. 1994; Green 2013; Si et al. 2014). While the connection has rarely been made in the literature, dC stars, having been rejuvenated by mass accretion, would likely be seen as blue stragglers if they were within a co-eval stellar cluster. They are also probably the dwarf progenitors of the typically more luminous carbon-enhanced metal-poor (CEMP), sgCH, $\mathrm{CH}$ and perhaps barium (Ba II) stars, which all show carbon and s-process enhancements (see discussion and references in De Marco \& Izzard 2017). The detection of white dwarf companions, and the characterization of the orbital properties of $\mathrm{dC}$ stars, is therefore important for understanding the mass transfer processes that give rise to this fascinating family of stars.

However, to date, the only dC star with a measured binary orbit is the prototype dC G77-61 (Dahn et al. 1977; Dearborn et al. 1986), a single-line spectroscopic binary (245 day period and semi-amplitude $20 \mathrm{~km} \mathrm{~s}^{-1}$ ), where the WD has cooled to $T_{\text {eff }}<6000 \mathrm{~K}$. Since G77-61 represents the only known dC with a proven radial velocity (RV) orbit, the mass-transfer hypothesis for dCs remains to be confirmed, and can be investigated only through the properties of dCs to detect and characterize host binary systems. Models for $\mathrm{dC}$ formation in both the disk and halo (de Kool \& Green 1995) predict a bimodal orbital period distribution, with a large peak at a $\sim$ decade (for accretion of the AGB wind at a binary separation $\sim 10 \mathrm{AU}$ ), and a smaller peak at $\sim$ year (for separations $\lesssim 1 \mathrm{AU}$ ) corresponding to systems that underwent a common envelope (CE) phase, where the companion was subsumed in the expanding atmosphere of the AGB star when it fills its Roche lobe. These models reproduce the better-studied distributions of $\mathrm{CH}$ and Ba II giants, whose progenitors are almost certainly the $\mathrm{dCs}$. The relic distribution of $\mathrm{dC}$ binary orbits should reveal the relative importance and efficiency of these types of accretion, which can substantially modify the $\mathrm{dC}$, leaving it hotter and bluer (and perhaps more rapidly rotating) than expected for its age.

Green (2013) identified 1220 faint $(r \gtrsim 17-21)$ C stars from SDSS spectra, $\sim 5$ times more than previously known, but also including a wider variety of $\mathrm{dC}$ properties than past techniques such as color or grism selection have netted. From those with significant proper motion measurements, they identified 730 definite dwarfs, including eight systems with clear DA white dwarf companions. This dataset represents the first significant sample of bona fide dCs appropriate for a population study.

The statistical analysis of large samples of sparsely sampled RV curves can be used to constrain the underlying properties (binary fraction and separation distribution) of the corresponding binary population (e.g., Maoz et al. 2012). The TDSS dwarf carbon star FES program will provide a second epoch of SDSS spectroscopy to measure RV variability for a large sample of $\mathrm{dC}$ stars, to produce first constraints on their binarity and the distribution of their orbital properties. The main aims of this program are to (i) test the binary evolution hypothesis for dwarf Carbon (dC) stars, (ii) constrain the distribution of orbital separations, and (iii) trace the chemistry and evolution of the oldest asymptotic-giant-branch (AGB) stars. The strategy used in the program will: (i) measure the RV shift $\Delta R V$ for $\mathrm{dC}$ stars between SDSS and TDSS (5-18 years), and (ii) constrain the separation distributions and hence the mass transfer mode.

For the dC FES program, we selected all 730 SDSS C stars from Green (2013) that were listed as dwarfs with high probability based on either their measured proper motions, or because they were identified from their SDSS spectra as composite DA/dC spectroscopic binaries. We added another $99 \mathrm{dC}$ stars found by Si et al. (2014), totalling 829 unique dC stars for repeat spectroscopy within TDSS. An example of SDSS archival and TDSS spectra of a dC in our program is shown in Figure 6.

\section{4. "Hypervariable" stars and quasars (TDSS_FES_HYPSTAR, TDSS_FES_HYPQSO)}

This program targets the most highly photometrically variable stars/classical pulsators (defined as hypervariable stars), as well as hypervariable quasars in the TDSS. The spectroscopic variability for these objects can potentially reveal large structural changes in astrophysical sources, and is useful for finding rare, transient phenomena such as "changing-look quasars" (e.g., LaMassa et al. 2015; Runnoe et al. 2016; Ruan et al. 2016b; MacLeod et al. 2016). More 


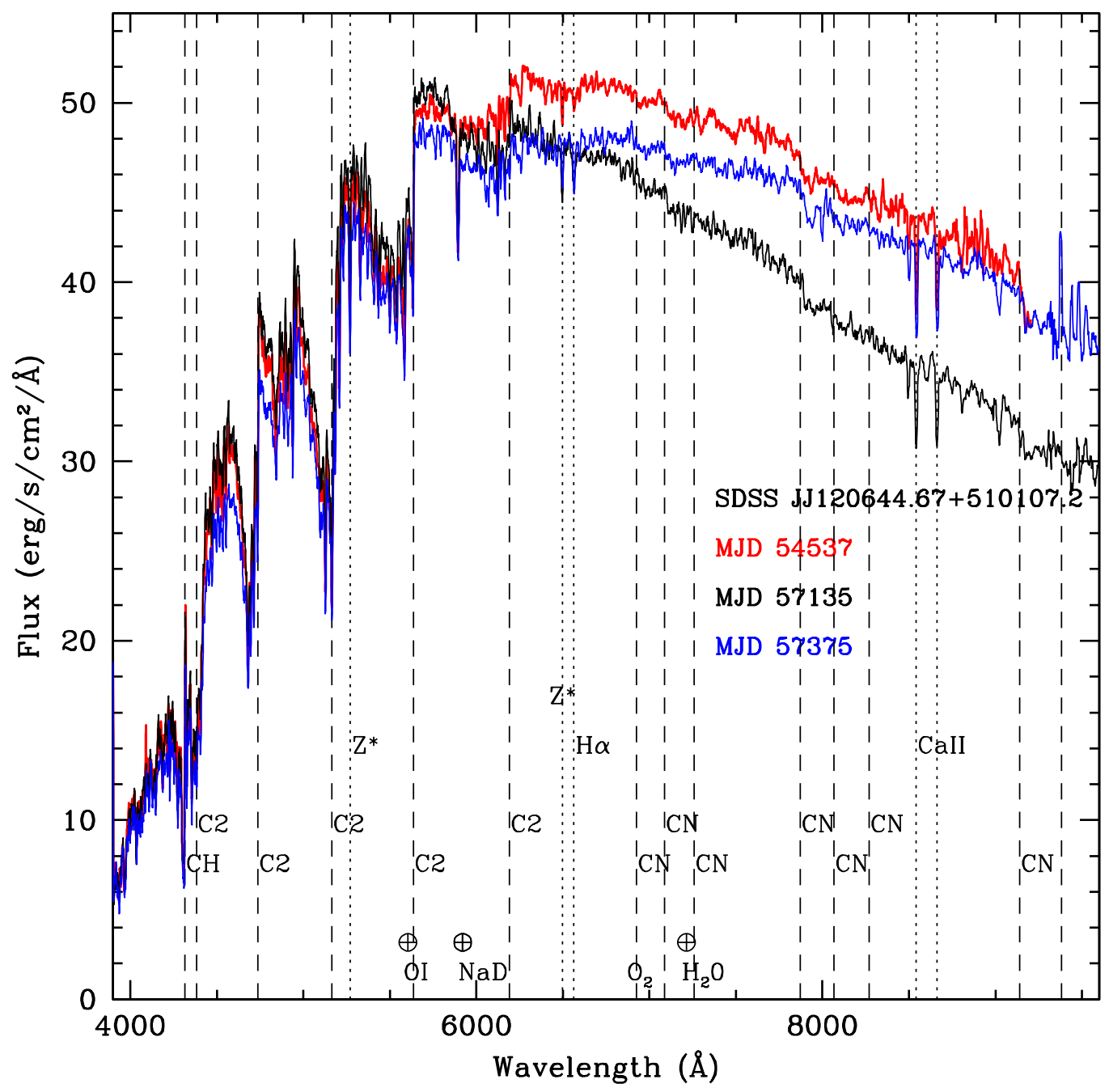

Figure 6. Example dwarf carbon star from TDSS (target class TDSS_FES_DWARFC, §3.3). The spectroscopic MJDS are 54537, 57135, and 57375. Prominent molecular bandheads of $\mathrm{CH}, \mathrm{C}_{2}$, and $\mathrm{CN}$ are labeled and marked by vertical dashed lines. Several other atomic features are marked with vertical dotted lines, including strong atomic metal line blends $\left(Z^{*}\right)$, H $\alpha$, and Ca II. The location of telluric features are marked across the bottom. TDSS allows study of RV variability, changes in color and brightness, and line strengths to illuminate the physics of these unique post mass transfer binaries.

importantly, this program is exploring unknown territory and therefore the scientific returns could be quite substantial.

During the variable target selection in the main TDSS SES program (Morganson et al. 2015), hypervariables are identified using a modified variability characterization that is designed to work in the extreme regions of variability space (see \$2.3). The hypervariable targets for these FES programs all have previous spectra in the SDSS DR11 SpecObjAll table. Since the pipeline classifications were adopted here without further verification, a few targeted stars may actually be quasars, and vice versa.

For stars, defined as $i<20$ point sources (uncorrected for Galactic extinction) with CLASS $=$ STAR, the top $0.5 \%$ most significantly variable objects were selected, corresponding approximately to $V>0.3 \mathrm{mag}$ (see Figure 7 ). These sources lie outside an approximately elliptical contour with SDSS-PS1 difference of $0.2 \mathrm{mag}$, a PS1-only variability of $0.15 \mathrm{mag}$, or some intermediate combination of the two (c.f. Figure 5, Morganson et al. 2015). The SDSS images of these sources are visually examined to remove objects with close neighbors, nearby diffraction spikes or other imaging issues that could significantly affect photometry. The above criteria select 1150 stars $\left(\sim 0.05 \mathrm{deg}^{-2}\right)$, which have the target flag TDSS_FES_HYPSTAR. Inspection of these targets' initial SDSS spectra suggest that this sample is rich in RR 


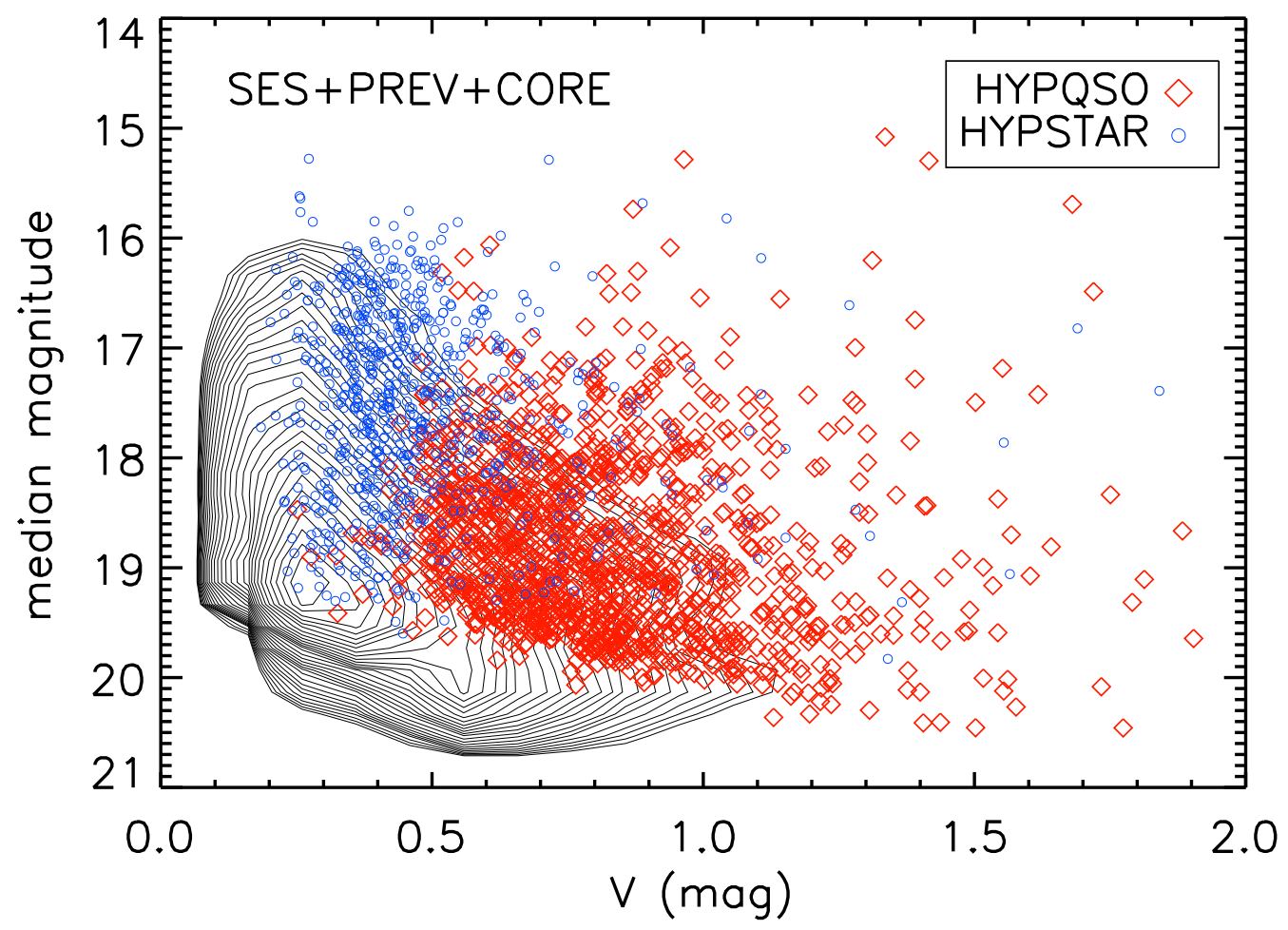

Figure 7. Distribution of the variability metric, $V$ (Eqn. 1), as a function of median magnitude among PS1 griz filters for all variability-selected quasars and stars described in Morganson et al. (2015). The logarithmic contours show the overall distribution for TDSS variables that either: a) are targeted for single epoch spectroscopy (SES) in TDSS, b) already have pre-existing spectra in SDSS (PREV), or c) are also targeted as part of the eBOSS CORE quasar program. The red diamonds (blue circles) show the distribution for hypervariable quasars (stars) targeted by the FES programs described in $\S 3.4$.

Lyrae variables and also includes M dwarfs, carbon stars and stars that are difficult to classify. For an example target, see Figure 8.

For quasars, defined as $i<20$ point sources (uncorrected for Galactic extinction) with CLASS $=$ QSO, the top $2 \%$ most significantly variable objects were selected, corresponding to approximately $V>0.5$ mag (see Figure 7 ). These sources lie outside an approximately elliptical contour with SDSS-PS1 difference of $0.7 \mathrm{mag}$, a PS1-only variability of $0.25 \mathrm{mag}$, or some intermediate combination of the two. The SDSS images of these sources are also visually examined to remove objects with close neighbors, nearby diffraction spikes or other imaging issues that could significantly affect photometry. The above criteria select 1555 quasars $\left(\sim 0.05 \mathrm{deg}^{-2}\right)$, which have the target flag TDSS_FES_HYPQSO. Inspection of these targets' initial SDSS spectra suggest that this sample is rich in BAL quasars and blazars, but otherwise contains a wide range of quasar types (we leave a detailed census to a later publication). For example spectra, see Figure 8.

\subsection{Broad absorption line variations in quasars (TDSS_FES_VARBAL)}

This FES program will build upon recent systematic, sample-based studies of BAL variability (e.g., Barlow 1993; Lundgren et al. 2007; Filiz Ak et al. 2012, 2013, 2014; Vivek et al. 2014, and references therein) by re-observing $~ 3000$ BAL quasars from SDSS and BOSS. About 2/3 of the sample was selected from Gibson et al. (2009) and has already been mostly observed as part of a BOSS ancillary proposal (see Filiz Ak et al. 2013) and probes rest-frame timescales of $\approx 4-7$ yr. TDSS is obtaining a third spectroscopic epoch for this subsample, typically spanning an additional 1$3 \mathrm{yr}$ in the rest frame beyond the most recent BOSS observations. The TDSS data yield improved measurements of the dependence of BAL EW variability upon rest-frame timescale, enabling a test of the extent to which long-term variability trends found in the SDSS-I/II vs. BOSS data persist. A third epoch also allows for the possibility of detecting BAL acceleration or re-emergence/disappearance. The long timescales sampled by this project are highly beneficial since velocity shifts associated with BAL acceleration/deceleration accumulate over time; the first results on this project's BAL acceleration are presented in Grier et al. (2016). Constraints upon BAL disappearance and 

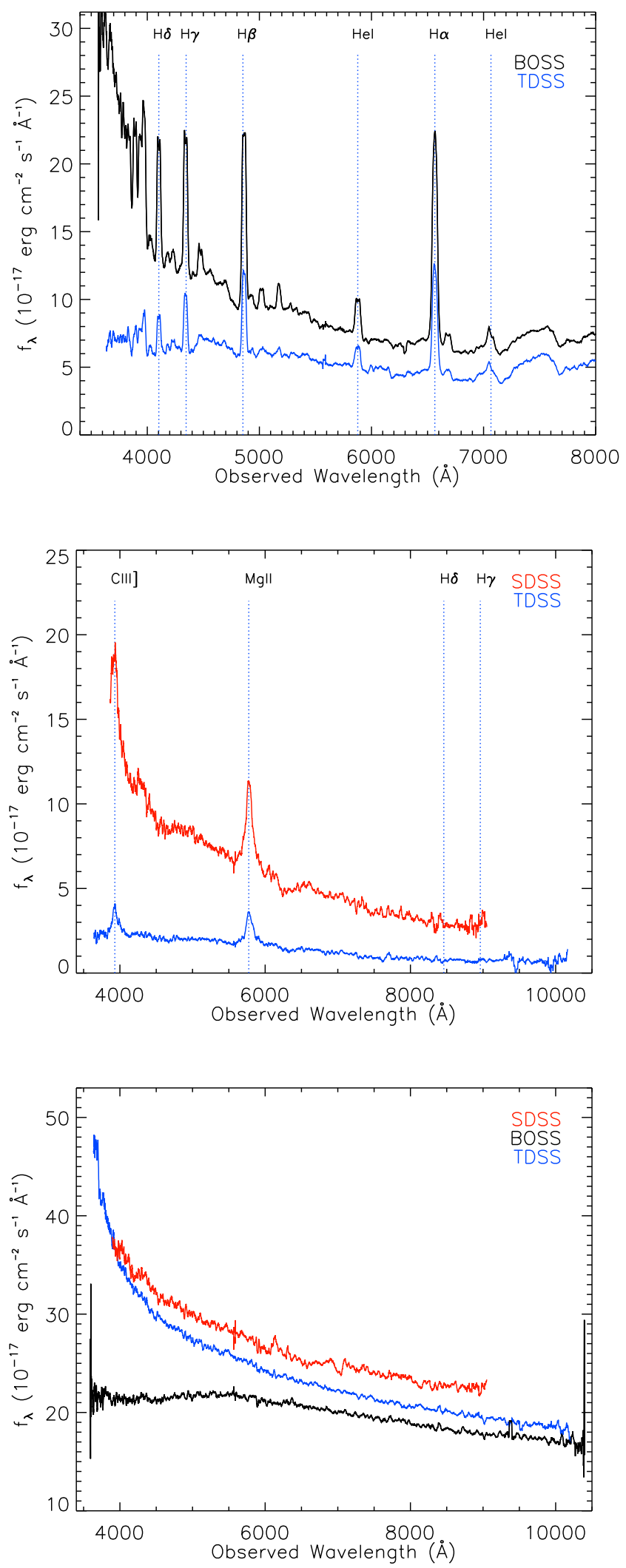

Figure 8. Examples of hypervariables (§3.4) targeted by the TDSS_FES_HYPSTAR (top panel), and TDSS_FES_HYPQSO (bottom two panels) programs. The object in the top panel is cataclysmic variable SDSS J003827.04+250925.0; the time between spectra is 2.9 yr. The middle panel shows hypervariable quasar SDSS J235040.09+002558.8 at redshift $z=1.062$, exhibiting a large change over $14.2 \mathrm{yr}$ (observed frame). Shown in the bottom panel is blazar SDSS J081815.99+422245.4 also found in Massaro et al. (2014), with spectroscopic MJDs 52205, 55505, and 57361 for SDSS, BOSS, and TDSS, respectively. 

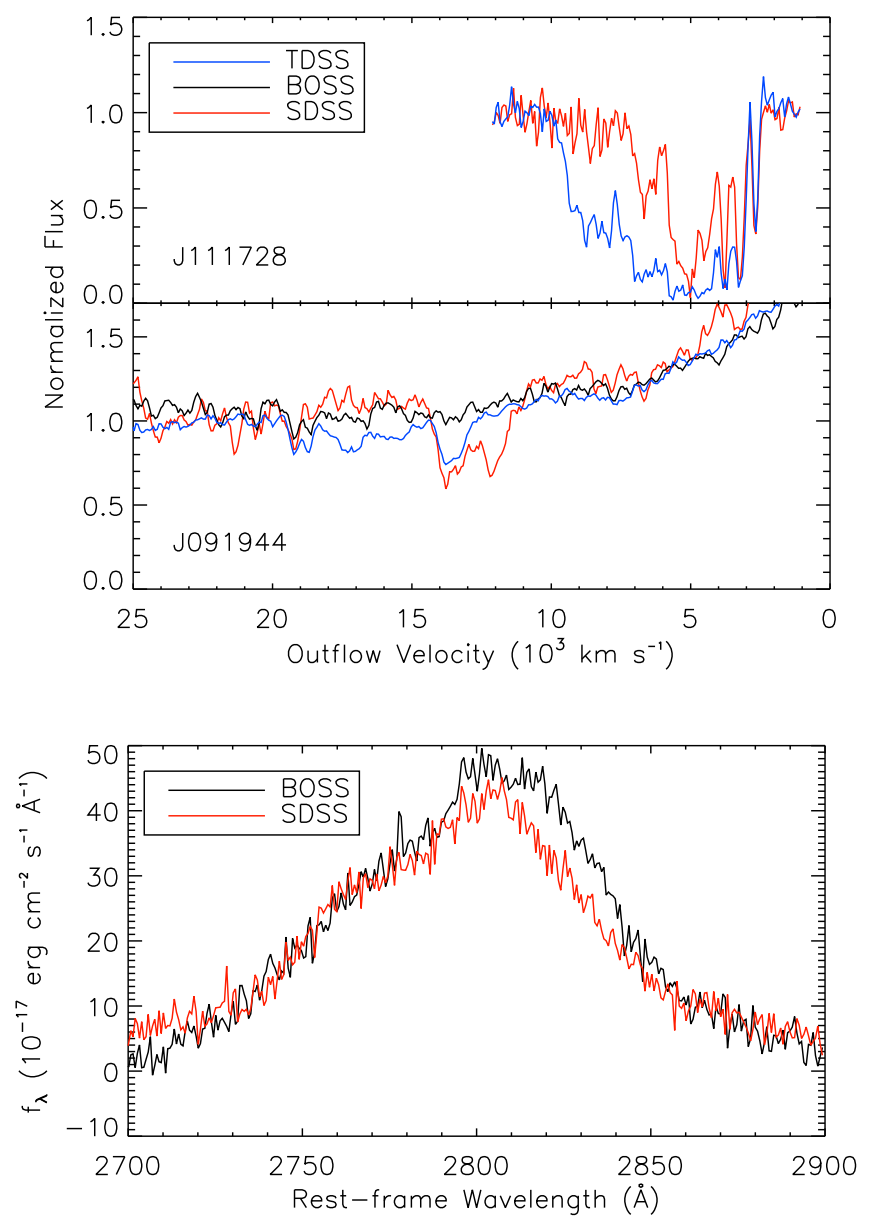

Figure 9. Top: Example spectra of BAL troughs from TDSS (target class TDSS_FES_VARBAL, $§ 3.5$ ). The C IV BAL troughs for quasars SDSS J111728.75+490216.4 (Grier et al. 2016) and SDSS J091944.53+560243.3 (McGraw et al. 2017) are displayed in the top and bottom panels respectively, where the velocity is relative to the rest frame wavelength of C IV. In the top panel, the spectroscopic MJDs are 57129 (TDSS) and 52438 (SDSS); in the bottom panel they are 57346 (TDSS), 56625 (BOSS), and 51908 (SDSS). The lower panel shows an example of a BAL re-emergence. Bottom: SDSS J163709.31+414030.8, a candidate SBHB at $z=0.760$ from Wang et al. (2017) showing a Mg II velocity shift similar to those in the target class TDSS_FES_MGII $(\S 3.6)$.

emergence provide key insights into the lifetime of BALs. Furthermore, BAL re-emergence events at the same velocity argue strongly against models where the variability is due to gas motions, instead favoring models where ionization changes play a key role. The first results on BAL re-emergence/disappearance are presented in McGraw et al. (2017). Finally, these observations further characterize the coordinated EW variations of BAL quasars with multiple troughs; these coordinated variations constrain models for BAL variability (e.g., Filiz Ak et al. 2012, 2013).

Figure 9 shows two examples of a variable BAL quasars observed in TDSS. The selection recipes used to obtain these targets are detailed below in a step-by-step manner.

\subsubsection{Main BAL sample}

The steps to select the majority of FES BAL quasars are as follows:

1. Match the DR5 BAL catalog (Gibson et al. 2009) to the DR5Q. This catalog provides full positional, photometric, and spectroscopic information for each BAL quasar. Positions agree to within 0.1 as expected. ${ }^{6}$ For the TDSS targeting, we adopt the astrometry as measured in SDSS DR9 (Ahn et al. 2012) for these objects.

2. Choose BAL quasars with $i<19.28$. These $i$ magnitudes in the DR5 quasar catalog are not corrected for

${ }^{6}$ Two quasars have different redshifts between the two catalogs: J100424.88+122922.2 and J153029.05+553247.9. These inconsistencies are explained on page 759, column 2 of Gibson et al. (2009). 
Galactic extinction, which is generally mild.

3. From the BAL quasars chosen in step 2, we only accept those with $\mathrm{BI}_{0}>100 \mathrm{~km} \mathrm{~s}^{-1}$ in one of their BAL troughs. Here, $\mathrm{BI}_{0}$ is the modified balnicity index defined in Gibson et al. (2009). This cut removes weak BALs that could have been mis-classified due to e.g., underlying continuum uncertainties.

We also constrain redshifts as follows (see Section 4 of Gibson et al. 2009): (i) 1.96-5.55 for Si IV BALs; (ii) 1.68-4.93 for C IV BALs; (iii) 1.23-3.93 for Al III BALs; and (iv) 0.48-2.28 for Mg II BALs. If a BAL quasar with troughs from multiple ions satisfies any one of these required redshift ranges, then it is accepted.

4. For the objects with coverage in the rest-frame window $1650-1750 \AA$, we only consider those with SNR_1700 $\geq 6$, where SNR_1700 is the SNR measurement in this wavelength window from the DR5 BAL catalog. This cut ensures a high-quality first-epoch spectrum for comparison purposes. The resulting number of BAL quasars is 2005.

5. At this point, a manual identification of 476 supplemental BAL targets was performed (led by author P. B. Hall). These targets may violate one or more of the above selection criteria, but have been identified as worthy of additional study nonetheless. ${ }^{7}$ They include the following object classes:

- BAL quasars originally detected in the Large Bright Quasar Survey (Hewett et al. 2001) or FIRST Bright Quasar Survey (White et al. 2000), or otherwise having discovery spectra predating SDSS by up to 10 years or more;

- Redshifted-Trough BAL quasars (Hall et al. 2013; Zhang et al. 2017), a rare class for which competing possible explanations make different predictions about trough variability;

- Overlapping-Trough BAL quasars with nearly complete absorption below Mg II at one epoch but which in several cases (Hall et al. 2011; Rafiee et al. 2016) have already shown extreme variability;

- BAL quasars observed more than once by SDSS and/or BOSS, and thus already possessing more than one epoch for comparison to SDSS-IV, including objects with BAL troughs which emerged between SDSS and BOSS;

- BAL or X-ray weak quasars selected for their unusual properties where observations of future variability (or lack thereof) may help determine the processes responsible for their unusual spectra.

After this addition, the resulting number of BAL quasars is 2481 (2005 regular plus 476 supplemental).

\subsubsection{DR12 Objects}

To increase the sky coverage of BAL targets, we employed a similar target selection as before for the BALs in a preliminary version of DR12Q from 22 March 2014 (I. Pâris 2014, private communication). To select BAL targets from this database, we focus only on C IV BAL selection, since this is arguably the primary ion of interest (and the one for which we had the needed data for selection). We require:

1. Magnitude $i<19.8$.

2. BI $>100 \mathrm{~km} \mathrm{~s}^{-1}$, where BI is the balnicity index defined by the parameter BI_CIV in Pâris et al. (2017).

3. BAL visual inspection flag to be positive (BAL_FLAG_VI $=1$; this cut only dismisses a few objects satisfying the BI_CIV > $100 \mathrm{~km} \mathrm{~s}^{-1}$ requirement and thus is a small effect).

4. A redshift range $z=1.68-4.93$, which provides complete coverage of the C IV BAL region.

5. The coordinates are within $-50^{\circ}<\alpha<50^{\circ}$ and $17.5^{\circ}<\delta<60^{\circ}$ (see above).

Application of the criteria above produces 294 targets.

Finally, a manual identification of 313 additional special BAL targets was performed (led by author P. B. Hall). These targets may violate one or more of the above selection criteria, but have been identified as critical for study nonetheless. These objects were selected in two different ways:

7 No explicit magnitude or SNR cut was made, but a very low SNR spectrum would have had to be quite interesting to be included. 
1. 307 quasars were selected from the preliminary DR12 quasar catalog. All targets have (BI_CIV $>0 \mathrm{~km} \mathrm{~s}^{-1}$ ) or (BAL_FLAG_VI $=1$ ) and one or more of the following: (i) OVI coverage (and preferentially narrow troughs); (ii) A high-velocity C IV trough (>30,000 $\mathrm{km} \mathrm{s}^{-1}$ ); (iii) Possible redshifted absorption; (iv) An existing SDSS spectrum as well as a BOSS spectrum; and $(v)$ Some other unusual property, thus classifying it as an "odd-BAL".

2. Six known quasars were selected from the printed catalogs of Junkkarinen et al. (1991, 1992) or Sowinski et al. (1997).

In total, thus, there are $294+313=607$ BAL quasars from this second pass of BAL targeting.

\subsection{Candidate Supermassive Binary Black Holes Based on Shifted Mg II Lines (TDSS_FES_MGII)}

Supermassive black hole binaries (SBHBs) are throught to be a common consequence of the merger of two massive galaxies. According to the evolutionary scenario described by Begelman et al. (1980), sometime after the merger of the parent galaxies, the two black holes form a bound binary whose separation decays first by dynamical friction, then by scattering of stars, and finally by the emission of gravitational radiation. ${ }^{8}$ The slowest stage in this evolutionary scheme is thought to correspond to an orbital separation of $0.01 \mathrm{pc} \lesssim a \lesssim 1 \mathrm{pc}$. Thus, observational efforts have focused on finding SBHBs at these orbital separations using RV variations of the broad emission lines (by analogy with double-lined or single-lined spectroscopic binary stars; e.g., Gaskell 1983, 1996). So far, direct observational evidence for SBHBs with two active BHs via this method has been elusive (e.g., Eracleous et al. 1997; Liu et al. 2016). Recent surveys have concentrated on candidates SBHBs with one active black hole and have utilized the large samples of quasar spectra available in the SDSS archive (Tsalmantza et al. 2011; Eracleous et al. 2012; Shen et al. 2013; Ju et al. 2013; Liu et al. 2014; Runnoe et al. 2017). The general strategy of these surveys is to select quasars whose broad Balmer or Mg II lines are offset from the frame defined by the narrow lines by $\sim 1000 \mathrm{~km} \mathrm{~s}^{-1}$ or more and/or search for systematic RV variations between the first-epoch spectra and spectra taken several years later.

This program is a continuation of the work of Ju et al. (2013) who studied the broad Mg II emission lines of $0.36<z<2$ quasars with multiple SDSS observations. The spectra from this program can be used to detect velocity shifts in SBHBs with separations of $\sim 0.1 \mathrm{pc}$ and orbital periods of $\sim 100$ years, assuming that the BHs have masses of order $10^{9} \mathrm{M}_{\odot}$.

From the sample of all quasars in DR7Q with multiple SDSS spectra of the Mg II line, Ju et al. (2013) identified seven robust SBHB candidates along with 57 more candidates that were less secure, for a total of 64 targets. The program is designed to obtain a third-epoch spectrum for all candidates, with highest priority given to the seven robust candidates, in order to search for monotonic velocity shifts relative to first epoch. The first results from this program were reported in Wang et al. (2017), in which the authors rule out a binary model for the bulk of candidates by comparing the variations in the velocity shifts over 1-2 yr and $10 \mathrm{yr}$. They also find that $\lesssim 1 \%$ of active SMBHs reside in binaries with $\sim 0.1 \mathrm{pc}$ separations observed in TDSS. The example shown in the bottom panel of Figure 9 is a candidate from Wang et al. (2017) with a prominent line shift.

\subsection{Variability of Disk-Like Broad Balmer Lines (TDSS_FES_DE)}

Broad Balmer lines with double-peaks, twin shoulders, or flat tops can be found in about $15 \%$ of radio-loud AGNs at $z<0.4$ (Eracleous \& Halpern 1994, 2003) and in about 3\% of AGNs at $z<0.33$ in the SDSS (Strateva et al. 2003), depending on radio-loudness and possibly Eddington ratio. Although a number of ideas have been discussed in the literature for the origin of these line profiles, a physical model attributing the emission to the outer parts of the accretion disk is the most successful in explaining the Balmer line profiles and other properties of these objects (see discussion in Eracleous \& Halpern 1994, 2003; Eracleous et al. 2009, and references therein). Thus, we refer to these objects as disk-like emitters hereafter. Previous long-term monitoring of disk-like emitters has sampled about two dozen objects over 20 years (e.g., Storchi-Bergmann et al. 2003; Gezari et al. 2007; Flohic 2008; Lewis et al. 2010; Popović et al. 2011, 2014; Sergeev et al. 2000, 2017, and references therein) at $z<0.4$, most of which are radio loud.

This FES program expands the scope of past monitoring efforts by re-observing for at least one more epoch a much larger number of disk-like emitters drawn from the SDSS. This selection method leads to a much wider variety of objects than those targeted by previous campaigns, namely more luminous objects, objects with higher Eddington ratios, and radio-quiet objects. This program also targets objects at $z \sim 0.6$, which are even more luminous than those at $z<0.4$.

8 There may be an additional phase before the emission of gravitational waves where the binary separation decays via interactions between the binary and a gaseous disk. 
Included in the target list are 1251 objects from DR7Q distributed over $\sim 6300 \mathrm{deg}^{2}$ (i.e., $0.2 \mathrm{deg}^{-2}$ ). The targets comprise "classic" disk-like emitters (at $z<0.33$ taken from Strateva et al. 2003) and higher-redshift analogs $(z \sim 0.6$; from Luo et al. 2013), as well as additional objects identified by Shen et al. (2011). A total of 220 objects are "classic" disk-like emitters (objects whose Balmer profiles can easily be modeled by a rotating accretion disk, e.g., Eracleous et al. 2009) while the remaining objects have very asymmetric Balmer profiles that can plausibly be attributed to a perturbed disk (for example one with a prominent spiral) or to a SBHB (see §3.6). The magnitudes of the targets are $i<18.9$. The TDSS spectra will cover $\mathrm{H} \alpha$ and $\mathrm{H} \beta$ for the $z<0.4$ objects, and $\mathrm{H} \beta$ and Mg II for the $z \sim 0.6$ objects. The time baseline will be $>10$ years for most objects. The 1251 targets of this program include 28 objects identified as promising sub-pc binary SMBH candidates with observed $\mathrm{H} \beta$ line shifts between two epochs in SDSS-I/II from Shen et al. (2013).

By combining existing SDSS spectra and spectra collected during TDSS, this program aims to address the following scientific goals. First, the observations will empirically characterize the variability of the broad emission line profiles, i.e., determine what property of the profiles is varying (e.g., width, asymmetry, shift, relative strengths and velocities of the peaks or shoulders), as well as the magnitude and time scale of the variations. Second, the data will be compared to a wide array of models of disk perturbations, including warps, self-gravitating clumps, and spiral or other waves. Third, this program aims to determine whether the variations represent systematic drifts of the line profiles and evaluate whether these changes are consistent with RV shifts due to orbital motion in a SBHB.

An example of disk-like emitter variability seen in one of the targets of this program is shown in the top panel of Figure 10.

\subsection{Variability of Broad Balmer Lines of Quasars With High-SNR Spectra (TDSS_FES_NQHISN)}

This program will yield second (or third) epoch spectra of bright, low-redshift $(z<0.8)$ SDSS quasars with existing high SNR spectra (requiring that the median SNR per spectral pixel across the full SDSS spectral range is $>23$ ). The combination of old and new spectra will be used to study the general broad line variability of quasars, including line shape changes and line centroid shifts, on multi-year timescales. The scientific goals are similar to those of the previous program (see §3.7). In addition to furthering our understanding of the dynamics of the gas in the broad-line region, the data from this program will be important for two more applications: (i) a comparison of the variability properties of typical quasar broad emission lines to the variability properties of disk-like emission lines (see §3.7), and (ii) selection of SBHB candidates via velocity shifts.

The focus of this program is quasars in DR7Q at $z<0.8$. Thus, the spectra will include the $\mathrm{H} \beta$ line, as well as the narrow [O III] doublet that will provide a reliable redshift and a velocity reference (e.g., Hewett \& Wild 2010). Included in this sample are 1486 quasars with a median SNR $>23$ per pixel.

For an example of a quasar targeted in this program, see Figure 10. This program is also producing serendipitous discoveries, for example the changing-look quasar fromRunnoe et al. (2016) was identified from NQHISN spectra.

\section{REPEAT QUASAR SPECTROSCOPY (RQS)}

Quasar variability on multi-year timescales is poorly characterized for large samples, and our efforts to date have produced unexpected and exciting results on the (dis)appearance of broad absorption and emission lines (e.g., Filiz Ak et al. 2012; Runnoe et al. 2016) as well as large variability of the continuum and broad line profile shapes. Clearly, in addition to continuing the existing TDSS programs, a more systematic investigation of quasar spectroscopic variability is warranted. As part of the eBOSS ELG survey (Raichoor et al. 2017), the TDSS was allotted a nominal target density of $10 \mathrm{deg}^{-2}$. As for previous plates, we reserve 10\% of TDSS fibers for the FES programs described in §3. For the remaining fibers, we target known quasars for an additional epoch of spectroscopy (therefore, no SES targets were included on the ELG plates). The target list includes a magnitude-limited sample of quasars to $i<19.1$, accounting for the majority of targets $\left(\gtrsim 7 \mathrm{deg}^{-2}\right)$, and a variability-selected subsample based on the light curve $\chi^{2}$, favoring quasars with highly-significant photometric variability. We also adopt the RQS target selection described here for the eBOSS plates covering the LRG/quasar targets within Thin82 (chunk20).

The RQS program is distinct from the SES TDSS target selection because it (i) targets known quasars for repeat spectroscopy so that spectroscopic variability can be studied, (ii) instead of a pure variability selection, includes a complete magnitude-limited sample, since the targets are already known to be quasars, (iii) uses the full SDSS+PS1 photometric variability information to populate fibers in the S82 region, and (iv) includes quasars with extended morphologies. Quasars with extended morphology are typically lower-luminosity, lower-redshift sources compared to the overall SDSS quasar sample, and they have been shown to display relatively larger variability amplitudes (Gallastegui-Aizpun \& Sarajedini 2014). In addition, by including morphologically extended quasars in RQS, we 

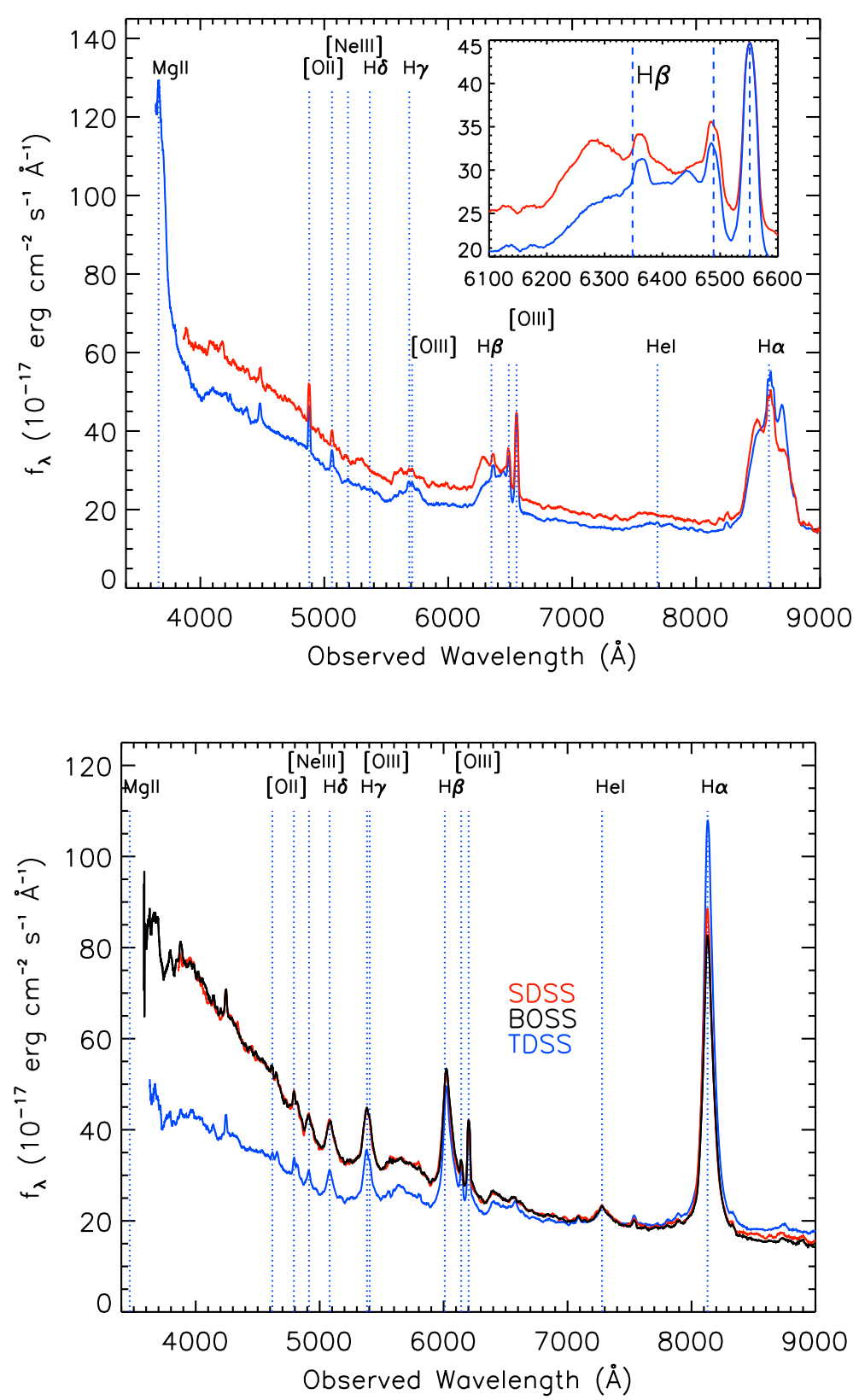

Figure 10. Top: SDSS J004319.74+005115.4, a disk-like emitter quasar at redshift $z=0.308$ (see $\S 3.7$ ) originally observed with the SDSS-I/II spectrograph (red) with an additional epoch of spectroscopy from TDSS (blue). The inset shows the area surrounding the $\mathrm{H} \beta$ emission line. This object has dramatic profile variations over 15 yr (observed frame) that may provide clues to the structure and dynamics of the BLR. Bottom: SDSS J011254.91+000313.0, a $z=0.238$ quasar observed at high SNR (see §3.8) that also has a spectrum in BOSS (shown in black and very similar to the SDSS spectrum). The TDSS and BOSS spectra (MJDs 57002 and 55214) have been scaled so the flux of [O III] matches that of the earlier SDSS spectrum (MJD 51794). The same level of smoothing has been applied to SDSS, BOSS, and TDSS spectra, and all have an effective wavelength $\lambda_{\text {eff }}=5400 \AA$.

increase the redshift/volume overlap with anticipated eROSITA AGN samples (Merloni et al. 2012). The RQS targets also include new SDSS-IV quasars (Myers et al. 2015; Palanque-Delabrouille et al. 2016), which, compared to the previous data releases, are on average fainter and extend out to higher redshifts.

The criteria and priorities $p$ for selecting quasars for repeat spectroscopy are first described broadly in the enumerated list below and then in more detail for each sky region. The FES targets make up the top-priority TDSS targets $(p=0)$ over the entire RQS footprint. For the remaining fibers with $p>0$, we identify quasars as follows: 
1. We start with all SDSS quasars drawn from DR7Q and DR12Q, and any new SDSS-IV objects with CLASS=QSO. Quasars are restricted to $17<i_{P S F}<21$, where $i_{P S F}$ is defined as the median SDSS PSF magnitude.

2. For the majority ${ }^{9}$ of the selection, our parent sample is quasars with at least two detections in both $g$ and $r$-bands among SDSS and PS1 data. To construct this parent sample, we consider all primary and secondary SDSS photometry within a $1^{\prime \prime}$ radius, along with PS1 magnitudes measured to better than $\sigma_{\mathrm{PS} 1}<0.15$ mag in $g_{\mathrm{PS} 1}$ and $r_{\mathrm{PS} 1}$, without regard to morphology or data quality flags.

3. Across all sky regions, the subset with $i_{P S F}<19.1$ defines our highest priority RQS targets $(p=1)$.

4. Also included in the top RQS priority class are $i_{P S F}<20.5$ quasars with multiple existing spectra $\left(N_{\text {spec }}>1\right)$, except for the region in Stripe 82 where the density for such repeatedly-observed objects exceeds the TDSS fiber density allotment. ${ }^{10}$

5. Within Stripe 82, the SDSS-IV footprint, and a part of the NGC, we use a variability selection to fill the remaining fibers. These lower-priority targets are defined by different cuts in $\chi_{p d f}^{2}$, the reduced $\chi^{2}$ for a model for which the quasar's brightness level does not vary (§2.3). The same cut is applied in both $g$ and $r$ bands.

Since the density of SDSS quasars varies greatly across the SDSS footprint, with S82 being the densest, we apply these different cuts depending on the sky region to achieve an approximately uniform final target density. The nonuniform coverage of SDSS-IV quasars also alters our selection method from field to field. After the $i<19.1$ selection (target flag TDSS_RQS1), we either use a variability or magnitude cut to fill the remaining target density depending on the sky region, where variability-selected targets have a "v" appended to the target flag (e.g., TDSS_RQS2v). ${ }^{11}$ A variability cut is especially useful in regions of high density since a magnitude cut would severly bias the selection to the brightest sources. Furthermore, the variability information is the best in the densest region (S82). Based on the final target densities, the bulk of the variability selected targets are in S82.

The target priorities are enumerated below for each region of the RQS footprint, where (1) is the highest RQS priority (with target flag TDSS_RQS1). By including objects marked TDSS_RQS2 or TDSS_RQS2v, we achieve a rather uniform surface density near the TDSS allotment of about $10 \mathrm{deg}^{-2}$, although we supplied targets at a higher density than the nominal $10 \mathrm{deg}^{-2}$ at lower priority to fill in any potential gaps in the ELG target density. ${ }^{12}$ In what follows, we adopt the J2000 coordinates from the DR10 PhotObj table:

- SGC ELG plates: Thin82 (chunk 21: $\left.317^{\circ}<\alpha<360^{\circ},-2^{\circ}<\delta<2^{\circ}\right)$ and Thick82 (chunk 22: $0<\alpha<45^{\circ}$, $\left.-5^{\circ}<\delta<5^{\circ}\right)$

- Region 1 (least dense): off of S82, and currently lacking SDSS-IV coverage (see Table A1)

All verified spectroscopic quasars with

1. $i_{P S F}<19.1$ or $>1$ existing spectra for $i_{P S F}<20.5$

2. $i_{P S F}<20.8$, which achieves a surface density near $11 \mathrm{deg}^{-2}$

3. $i_{P S F}<21$, which achieves a surface density near $15 \mathrm{deg}^{-2}$

- Region 2: off S82 with SDSS-IV coverage:

All verified spectroscopic quasars with

1. $i_{P S F}<19.1$ or $>1$ existing spectra for $i_{P S F}<20.5$

2. $\chi_{\text {pdf }}^{2}>27$ for $i_{P S F}<20.5$ to achieve $11 \mathrm{deg}^{-2}$

3. $\chi_{p d f}^{2}>15$ for $i_{P S F}<20.5$ to achieve $15 \mathrm{deg}^{-2}$

- Region 3 (most dense, $|\delta|<1.3^{\circ}$, in S82):

All verified spectroscopic quasars with

1. $i_{P S F}<19.1$

\footnotetext{
${ }^{9}$ In the NGC region with $126^{\circ}<\alpha \leq 133^{\circ}$, the full list of DR7-12 quasars, regardless of the number of photometric detections, forms our parent sample.

10 The density of $i<20.5$ quasars with $N_{\text {spec }}>1$ is $12.75 \mathrm{deg}^{-2}$ and $1 \mathrm{deg}^{-2}$ on and off Stripe 82, respectively.

11 This choice in target flags was made in order to distinguish between second-priority magnitude- and variability-selected targets on ELG plates that could potentially include both target types. Note that for the Thin82 plates in eBOSS chunk 20, the variability-selected targets have flag TDSS_RQS2.

12 If ELG targets are dense in a particular region, we might achieve somewhat less than $10 \mathrm{deg}^{-2}$. For regions where ELG targets are more sparse, TDSS can exceed its nominal $10 \mathrm{deg}^{-2}$ density by using targets labeled TDSS_RQS3 or TDSS_RQS3v, which achieve densities up to $15 \mathrm{deg}^{-2}$. We do not supply TDSS_RQS3 targets for the Thin82 plates in eBOSS chunk 20, as all submitted TDSS targets in that area receive a fiber.
} 
2. $\chi_{p d f}^{2}>57$ for $i_{P S F}<20.5$ to achieve $11 \mathrm{deg}^{-2}$

3. $\chi_{p d f}^{2}>33$ for $i_{P S F}<20.5$ to achieve $15 \mathrm{deg}^{-2}$

- Thin82 (chunk 20: $315^{\circ}<\alpha<360^{\circ},-2^{\circ}<\delta<2.75^{\circ}$ ):

1. $i_{P S F}<19.1$

2. $\chi_{p d f}^{2}>57$, which achieves a surface density near $11 \mathrm{deg}^{-2}$

- NGC ELG plates (with $376 \mathrm{deg}^{2}$ already tiled: two rectangles spanning $126^{\circ}<\alpha<142.5^{\circ}, 16^{\circ}<\delta<29^{\circ}$; and $\left.137^{\circ}<\alpha<157^{\circ}, 13.8^{\circ}<\delta<27^{\circ}\right)$

- For $133^{\circ}<\alpha<142.5^{\circ}, 16^{\circ}<\delta<29^{\circ}$; and $137^{\circ}<\alpha<157^{\circ}, 13.8^{\circ}<\delta<27^{\circ}$ :

1. $i_{P S F}<19.1$ or $>1$ existing spectra for $i_{P S F}<20.5$ : reaches $10 \mathrm{deg}^{-2}$

2. $\chi_{\text {pdf }}^{2}>15$ for $i_{P S F}<20.5$ to achieve $12 \mathrm{deg}^{-2}$

3. $\chi_{p d f}^{2}>2$ for $i_{P S F}<20.5$ to achieve $17 \mathrm{deg}^{-2}$

- For all other NGC regions:

1. $i_{P S F}<19.1$ : reaches $10 \mathrm{deg}^{-2}$

2. $>1$ existing spectra for $i_{P S F}<20.5$ : reaches $12 \mathrm{deg}^{-2}$

3. $i_{P S F}<20$ : reaches $17 \mathrm{deg}^{-2}$

All new SDSS-IV objects selected by the above criteria are visually confirmed as quasars by inspecting the eBOSS spectra. We find that this step was mainly necessary for those quasars with the ZWARNING flag set, but we inspect all selected SDSS-IV objects regardless. For objects selected based on $\chi_{p d f}^{2}$, we have visually inspected a $3^{\prime} \times 3^{\prime}$ SDSS image and rejected objects with close (less than about $5^{\prime \prime}$ ) neighbors of similar brightness, as well as objects with nearby bright stars (or extended galaxies) whose diffraction spikes or isophotes might reasonably contaminate the quasar's photometry. This image inspection removed about $4-8 \%$ of candidate targets, depending on the magnitude range.

The approximate anticipated time baselines for RQS targets with $p=1-2$ are shown in Figure 3. The recently confirmed SDSS-IV quasars extend the distribution of probed timescales to much shorter baselines. The final distribution of all $i<19, p=1$ RQS targets $(\approx 11,000$ quasars $)$ in rest-frame time baseline-luminosity plane is shown in Figure 4 . RQS is expected to fill in the large gaps in this plane. The number of targets in each RQS sky region are listed along with the numbers for the FES programs in Table 1, and an example RQS target observed in the Fall of 2016 is shown in Figure 11.

\section{SUMMARY}

We have described each sample that is targeted for spectroscopic reobservations in TDSS. These targets include the FES samples, containing stars and quasars of astrophysical interest, and the RQS sample, containing a comprehensive set of quasars. For the single-epoch "SES" TDSS target selection which constitutes the main TDSS sample, see Morganson et al. (2015); for initial results see Ruan et al. (2016a). By design, the FES programs total to an average target density of $1 \mathrm{deg}^{-2}$ throughout the TDSS survey area (10\% of all TDSS targets), whereas the RQS targets are prioritized to achieve a density of $\sim 10 \mathrm{deg}^{-2}$ over a smaller area $\left(\sim 1200 \mathrm{deg}^{2}\right)$. Observations of the SES and FES programs began in July 2014, and observations of the RQS targets started Fall 2016. TDSS observations are planned to continue until mid-2020, as part of SDSS-IV eBOSS (see Blanton et al. 2017). By the end of the survey, TDSS will have obtained reasonable statistical samples for each FES subclass, containing at least 100 to 1000 sources each, in addition to the $\sim 13,000$ new quasar spectra obtained by the RQS program (see Table 1 ).

The FES programs span a large range of scientific goals, including:

- Tracking magnetic field changes over weeks to months to decade-long timescales in $\mathrm{M}$ and $\mathrm{L}$ dwarfs through observed $\mathrm{H} \alpha$ variability $(\S 3.1)$;

- Investigating the effect of rotation and orbital parameters on stellar activity via the $\mathrm{H} \alpha \mathrm{EW}$ in white dwarf-M dwarf binaries (§3.2);

- Testing the binary evolution hypothesis for dwarf Carbon stars through RV shifts (§3.3); 
Table 1. Breakdown of FES/RQS Targets

\begin{tabular}{|c|c|c|c|}
\hline Target flag & Description & $\begin{array}{l}\text { No. targets } \\
\text { submitted }\end{array}$ & $\begin{array}{c}\text { No. targets } \\
\text { observed } \\
\text { as of DR14 }\end{array}$ \\
\hline TDSS_RQS1 ${ }^{\mathrm{a}}$ SGC (chunks $\left.21+22\right)$ & Repeat Quasar Spectroscopy on ELG plates & $828+3332$ & 0 \\
\hline TDSS_RQS2(v) ${ }^{\mathrm{b}}$ SGC (chunks 21+22) & Repeat Quasar Spectroscopy on ELG plates & $527+967$ & 0 \\
\hline TDSS_RQS3(v) ${ }^{\mathrm{c}}$ SGC (chunks 21+22) & Repeat Quasar Spectroscopy on ELG plates & $290+667$ & 0 \\
\hline TDSS_RQS1 ${ }^{\mathrm{a}}$ NGC (chunk 23) & Repeat Quasar Spectroscopy on ELG plates & 3366 & 0 \\
\hline TDSS_RQS2(v) ${ }^{\mathrm{b}}$ NGC (chunk 23) & Repeat Quasar Spectroscopy on ELG plates & 385 & 0 \\
\hline TDSS_RQS3(v) ${ }^{\mathrm{c}}$ NGC (chunk 23) & Repeat Quasar Spectroscopy on ELG plates & 845 & 0 \\
\hline TDSS_RQS1 ${ }^{\mathrm{a}}$ thin82 (chunk 20$)$ & Repeat Quasar Spectroscopy on LRG/QSO plates & 1064 & 0 \\
\hline TDSS_RQS2 ${ }^{\mathrm{b}}$ thin82 (chunk 20$)$ & Repeat Quasar Spectroscopy on LRG/QSO plates & 1069 & 0 \\
\hline TDSS_FES_DWARFC & Dwarf Carbon stars $(\S 3.3)$ & 830 & 125 \\
\hline TDSS_FES_WDDM & White dwarf/M dwarf pairs with $\mathrm{H} \alpha(\S 3.2)$ & 402 & 54 \\
\hline TDSS_FES_ACTSTAR & Late-type stars with $\mathrm{H} \alpha(\S 3.1)$ & 1036 & 156 \\
\hline TDSS_FES_HYPSTAR & Strongly variable stars $(\S 3.4)$ & 1180 & 215 \\
\hline TDSS_FES_VARBAL & Broad absorption line QSOs $(\S 3.5)$ & 3032 & 950 \\
\hline TDSS_FES_DE & QSO disk-like emitters $(\S 3.7)$ & 1251 & 232 \\
\hline TDSS_FES_MGII & Binary AGN candidates $(\S 3.6)$ & 64 & 27 \\
\hline TDSS_FES_NQHISN & QSOs with previous spectral SNR > 25 (§3.8) & 1486 & 324 \\
\hline TDSS_FES_HYPQSO & Strongly variable QSOs $(\S 3.4)$ & 1692 & 364 \\
\hline
\end{tabular}

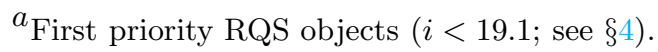

${ }^{b}$ Second priority RQS object (may or may not be variability selected).

${ }^{c}$ Third priority RQS object (may or may not be variability selected).

Note-Some target groups may overlap. DR14 contains data through May 11 2016. The RQS numbers are restricted to targets already assigned to fibers in the $996 \mathrm{deg}^{2}$ tiled area of the ELG survey so far - the ELG survey is planned to cover an additional $224 \mathrm{deg}^{2}$ in the NGC by the end of the fourth year of SDSS-IV. All targets are drilled at an effective wavelength of $\lambda_{\text {eff }}=5400 \AA$. Less than $10 \%$ of spectra are expected to be of poor quality.

- Exploring the hypervariable star and quasar populations (§3.4);

- Constraining models for BAL variability in quasars through analysis of BAL EW and profile variations over rest-frame timescales of $\approx 4-10 \mathrm{yr}$, including BAL acceleration and re-emergence/disappearance events $(\S 3.5)$;

- Searching for velocity shifts in SBHBs with separations of $\sim 0.1 \mathrm{pc}$ and orbital periods of $\sim 100$ years $(\S 3.6)$;

- Empirically characterizing the variability of broad emission line profiles in disk-like emitting quasars, informing models of disk perturbations (§3.7); and

- Studying the general broad line variability of quasars on multi-year timescales (§3.8).

The RQS program, comprising the bulk of the TDSS selection in the ELG and Thin82 plates, is intended to provide spectroscopic variability measurements for an unbiased, high-quality quasar sample that covers a wide range of redshift and luminosity. This dataset will form a complementary sample to the existing quasar sample with repeat spectroscopy from SDSS and BOSS, which contains 70K quasars as of DR14 (Paris et al. 2017), and will span a larger range of timescales. Along with sampling long timescales, an important aspect of the FES and RQS programs is that repeat spectra obtained within SDSS-IV, made available for the most part by the ELG survey (Raichoor et al. 2017), will sample short timescales that are also of astrophysical interest. 


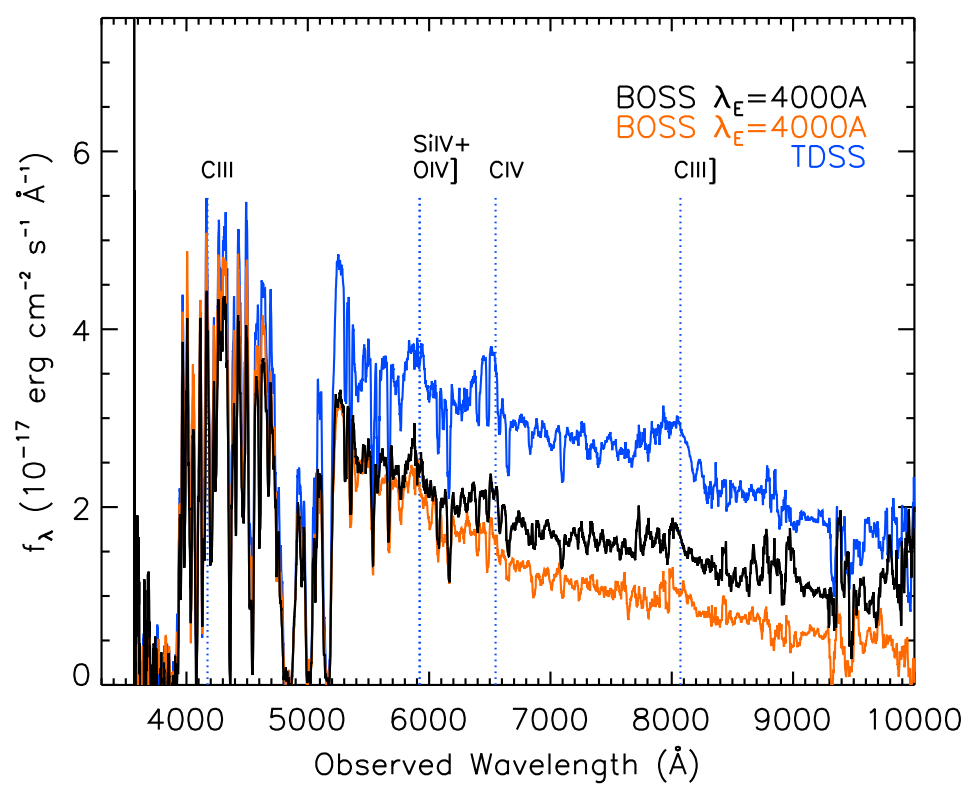

Figure 11. Example spectra of a RQS target observed by TDSS on MJD 57668 (target class TDSS_RQS1, §4). The quasar source has four intervening damped Lyman- $\alpha$ systems (Srianand et al. 2016). The two previous BOSS spectra of this object are shown, where that shown in orange (black) was taken on MJD 55499 (55505).

All the raw and reduced data will be released to the public as part of the main SDSS-IV data releases. The spectroscopic variability studies addressed in these programs will improve our understanding of the variable sky and facilitate our preparation for future sky surveys in the time domain, such as Pan-STARRS-2 (Burgett 2012), the Zwicky Transient Facility (Bellm 2014), and the Large Synoptic Survey Telescope (LSST Science Collaboration et al. 2009). Additionally, the samples presented here will help establish the targeting strategy for future large-scale spectroscopic variability studies currently planned for after SDSS-IV.

We thank an anonymous referee whose comments greatly improved the manuscript. We also thank Lile Wang for provided the data needed for the bottom panel of Figure 9. Support for this work was provided by the National Aeronautics and Space Administration through Chandra Award Number AR4-15016X issued by the Chandra X-ray Observatory Center, which is operated by the Smithsonian Astrophysical Observatory for and on behalf of the National Aeronautics Space Administration under contract NAS8-03060. This material is based upon work supported in part by the National Science Foundation under Grants AST-1715121 and AST-1715763. WNB, CJG, and SM acknowledge support from NSF grant AST-1516784. YS acknowledges support from an Alfred P. Sloan Research Fellowship and NSF grant AST-1715579. Funding for the Sloan Digital Sky Survey IV has been provided by the Alfred P. Sloan Foundation, the U.S. Department of Energy Office of Science, and the Participating Institutions. SDSS acknowledges support and resources from the Center for High-Performance Computing at the University of Utah. The SDSS web site is www.sdss.org.

SDSS is managed by the Astrophysical Research Consortium for the Participating Institutions of the SDSS Collaboration including the Brazilian Participation Group, the Carnegie Institution for Science, Carnegie Mellon University, the Chilean Participation Group, the French Participation Group, Harvard-Smithsonian Center for Astrophysics, Instituto de Astrofísica de Canarias, The Johns Hopkins University, Kavli Institute for the Physics and Mathematics of the Universe (IPMU) / University of Tokyo, Lawrence Berkeley National Laboratory, Leibniz Institut für Astrophysik Potsdam (AIP), Max-Planck-Institut für Astronomie (MPIA Heidelberg), Max-Planck-Institut für Astrophysik (MPA Garching), Max-Planck-Institut für Extraterrestrische Physik (MPE), National Astronomical Observatories of China, New Mexico State University, New York University, University of Notre Dame, Observatrio Nacional / MCTI, The Ohio State University, Pennsylvania State University, Shanghai Astronomical Observatory, United Kingdom Participation Group, Universidad Nacional Autnoma de México, University of Arizona, University of Colorado Boulder, University of Oxford, University of Portsmouth, University of Utah, University of Virginia, University of Washington, University of Wisconsin, Vanderbilt University, and Yale University. 
Table A1. SDSS-IV Coverage as of Summer 2016

\begin{tabular}{|c|c|}
\hline $\mathrm{RA}\left({ }^{\circ}\right)$ & $\operatorname{Dec}\left({ }^{\circ}\right)$ \\
\hline \multicolumn{2}{|c|}{ Boxes spanning ranges: } \\
\hline $348<\alpha<357$ & $-1<\delta<+1$ \\
\hline$\alpha>357$ & $-3.5<\delta<+7.2$ \\
\hline $8.2<\alpha<19.4$ & $-8.6<\delta<-5.9$ \\
\hline $0.0<\alpha \leq 32.8$ & $-5.6<\delta<+3.1$ \\
\hline $32.8<\alpha<45.0$ & $-6.0<\delta<+3.1$ \\
\hline $0.0<\alpha<1.75$ & $+4.6<\delta<+7.0$ \\
\hline \multicolumn{2}{|c|}{ Circles of radius $1.5^{\circ}$ centered on: } \\
\hline 4.3 & +4.0 \\
\hline 6.8 & +4.0 \\
\hline 14.5 & +4.0 \\
\hline 30.0 & +4.0 \\
\hline 36.7 & +4.0 \\
\hline 42.3 & +4.0 \\
\hline
\end{tabular}

The Pan-STARRS1 Surveys (PS1) and the PS1 public science archive have been made possible through contributions by the Institute for Astronomy, the University of Hawaii, the Pan-STARRS Project Office, the Max-Planck Society and its participating institutes, the Max Planck Institute for Astronomy, Heidelberg and the Max Planck Institute for Extraterrestrial Physics, Garching, The Johns Hopkins University, Durham University, the University of Edinburgh, the Queen's University Belfast, the Harvard-Smithsonian Center for Astrophysics, the Las Cumbres Observatory Global Telescope Network Incorporated, the National Central University of Taiwan, the Space Telescope Science Institute, the National Aeronautics and Space Administration under Grant No. NNX08AR22G issued through the Planetary Science Division of the NASA Science Mission Directorate, the National Science Foundation Grant No. AST-1238877, the University of Maryland, Eotvos Lorand University (ELTE), the Los Alamos National Laboratory, and the Gordon and Betty Moore Foundation.

\section{Facilities: SDSS, Pan-STARRS}

\section{APPENDIX}

\section{A. SDSS-IV COVERAGE}

Table A1 lists the regions of sky relevant to the ELG survey (Raichoor et al. 2017) that had SDSS-IV coverage at the time of RQS targeting. This information would be necessary for reproducing the RQS target selection.

\section{REFERENCES}

Abazajian, K. N., Adelman-McCarthy, J. K., Agüeros, M. A., et al. 2009, ApJS, 182, 543

Abolfathi, B., Aguado, D. S., Aguilar, G., et al. 2017, ArXiv e-prints, arXiv:1707.09322

Ahn, C. P., Alexandroff, R., Allende Prieto, C., et al. 2012, ApJS, 203, 21

Aihara, H., et al. 2011, The Astrophysical Journal Supplement Series, 193, 29

Alam, S., Albareti, F. D., Allende Prieto, C., et al. 2015, ApJS, 219, 12

Anderson, S. F., Voges, W., Margon, B., et al. 2003, AJ, 126, 2209

Barlow, T. A. 1993, PhD thesis, California University

Barth, A. J., Voevodkin, A., Carson, D. J., \& Woźniak, P. 2014, AJ, 147, 12

Becker, R. H., White, R. L., \& Helfand, D. J. 1995, ApJ, 450, 559
Begelman, M. C., Blandford, R. D., \& Rees, M. J. 1980, Nature, 287, 307

Bellm, E. 2014, in The Third Hot-wiring the Transient Universe Workshop, ed. P. R. Wozniak, M. J. Graham, A. A. Mahabal, \& R. Seaman, 27-33

Berger, E., Rutledge, R. E., Phan-Bao, N., et al. 2009, ApJ, 695, 310

Blanton, M. R., Bershady, M. A., Abolfathi, B., et al. 2017, AJ, 154,28

Burgett, W. S. 2012, in Proc. SPIE, Vol. 8449, Modeling, Systems Engineering, and Project Management for Astronomy $\mathrm{V}, 84490 \mathrm{~T}$

Butler, N. R., \& Bloom, J. S. 2011, AJ, 141, 93

Cackett, E. M., Gültekin, K., Bentz, M. C., et al. 2015, ApJ, 810,86 
Dahn, C. C., Liebert, J., Kron, R. G., Spinrad, H., \& Hintzen, P. M. 1977, ApJ, 216, 757

Davenport, J. R. A. 2016, ApJ, 829, 23

Dawson, K., et al. 2013, AJ, 145, 10

Dawson, K. S., Kneib, J.-P., Percival, W. J., et al. 2016, AJ, 151, 44

de Kool, M., \& Green, P. J. 1995, ApJ, 449, 236

De Marco, O., \& Izzard, R. G. 2017, PASA, 34, e001

Dearborn, D. S. P., Liebert, J., Aaronson, M., et al. 1986, ApJ, 300,314

Doi, M., Tanaka, M., Fukugita, M., et al. 2010, AJ, 139, 1628

Drake, A. J., Graham, M. J., Djorgovski, S. G., et al. 2014, ApJS, 213, 9

Eisenstein, D. J., Weinberg, D. H., Agol, E., et al. 2011, AJ, 142, 72

Eracleous, M., Boroson, T. A., Halpern, J. P., \& Liu, J. 2012, ApJS, 201, 23

Eracleous, M., \& Halpern, J. P. 1994, ApJS, 90, 1

—. 2003, ApJ, 599, 886

Eracleous, M., Halpern, J. P., M. Gilbert, A., Newman, J. A., \& Filippenko, A. V. 1997, ApJ, 490, 216

Eracleous, M., Lewis, K. T., \& Flohic, H. M. L. G. 2009, NewAR, 53, 133

Filiz Ak, N., Brandt, W. N., Hall, P. B., et al. 2012, ApJ, 757, 114

-. 2013, ApJ, 777, 168

—. 2014, ApJ, 791, 88

Flewelling, H. A., Magnier, E. A., Chambers, K. C., et al. 2016, ArXiv e-prints, arXiv:1612.05243

Flohic, H. M. L. 2008, PhD thesis, The Pennsylvania State University

Frieman, J. A., Bassett, B., Becker, A., et al. 2008, AJ, 135, 338

Fukugita, M., Ichikawa, T., Gunn, J. E., et al. 1996, AJ, 111, 1748

Gallastegui-Aizpun, U., \& Sarajedini, V. L. 2014, MNRAS, 444, 3078

Gaskell, C. M. 1983, in Liege International Astrophysical Colloquia, Vol. 24, Liege International Astrophysical Colloquia, ed. J.-P. Swings, 473-477

Gaskell, C. M. 1996, in Lecture Notes in Physics, Berlin Springer Verlag, Vol. 471, Jets from Stars and Galactic Nuclei, ed. W. Kundt, 165

Gezari, S., Halpern, J. P., \& Eracleous, M. 2007, ApJS, 169, 167

Gibson, R. R., Jiang, L., Brandt, W. N., et al. 2009, ApJ, 692, 758

Gizis, J. E., Reid, I. N., \& Hawley, S. L. 2002, AJ, 123, 3356

Green, P. 2013, ApJ, 765, 12

Green, P. J., Margon, B., Anderson, S. F., \& MacConnell, D. J. 1992, ApJ, 400, 659

Grier, C. J., Brandt, W. N., Hall, P. B., et al. 2016, ApJ, 824, 130

Gunn, J. E., Carr, M., Rockosi, C., et al. 1998, AJ, 116, 3040

Gunn, J. E., Siegmund, W. A., Mannery, E. J., et al. 2006, AJ, 131,2332

Guo, H., \& Gu, M. 2016, ApJ, 822, 26

Hall, P. B. 2002, ApJL, 564, L89

Hall, P. B., Anosov, K., White, R. L., et al. 2011, MNRAS, 411, 2653

Hall, P. B., Brandt, W. N., Petitjean, P., et al. 2013, MNRAS, 434,222

Harris, D. W., Jensen, T. W., Suzuki, N., et al. 2016, AJ, 151, 155

Heber, U., Bade, N., Jordan, S., \& Voges, W. 1993, A\&A, 267, L31

Hewett, P. C., Foltz, C. B., \& Chaffee, F. H. 2001, AJ, 122, 518

Hewett, P. C., \& Wild, V. 2010, MNRAS, 405, 2302

Iben, Jr., I., \& Renzini, A. 1983, ARA\&A, 21, 271
Inserra, C., Smartt, S. J., Jerkstrand, A., et al. 2013, ApJ, 770, 128

Ivezić, Ž., Smith, J. A., Miknaitis, G., et al. 2007, AJ, 134, 973

Ju, W., Greene, J. E., Rafikov, R. R., Bickerton, S. J., \& Badenes, C. 2013, ApJ, 777, 44

Junkkarinen, V., Hewitt, A., \& Burbidge, G. 1991, ApJS, 77, 203

-. 1992, ApJS, 81, 409

Kaiser, N., Aussel, H., Burke, B. E., et al. 2002, in Proc. SPIE, Vol. 4836, Survey and Other Telescope Technologies and Discoveries, ed. J. A. Tyson \& S. Wolff, 154-164

Kaiser, N., et al. 2010, in Society of Photo-Optical Instrumentation Engineers (SPIE) Conference Series, Vol. 7733, Society of Photo-Optical Instrumentation Engineers (SPIE) Conference Series, 0

Korista, K. T., \& Goad, M. R. 2004, ApJ, 606, 749

LaMassa, S. M., Cales, S., Moran, E. C., et al. 2015, ApJ, 800, 144

Lewis, K. T., Eracleous, M., \& Storchi-Bergmann, T. 2010, ApJS, 187, 416

Liebert, J., Schmidt, G. D., Lesser, M., et al. 1994, ApJ, 421, 733

Liu, J., Eracleous, M., \& Halpern, J. P. 2016, ApJ, 817, 42

Liu, X., Shen, Y., Bian, F., Loeb, A., \& Tremaine, S. 2014, ApJ, 789,140

LSST Science Collaboration, Abell, P. A., Allison, J., et al. 2009, ArXiv e-prints, arXiv:0912.0201

Lundgren, B. F., Wilhite, B. C., Brunner, R. J., et al. 2007, ApJ, 656,73

Lundgren, B. F., Brunner, R. J., York, D. G., et al. 2009, ApJ, 698,819

Luo, B., Brandt, W. N., Eracleous, M., et al. 2013, MNRAS, 429,1479

MacLeod, C. L., Ross, N. P., Lawrence, A., et al. 2016, MNRAS, 457,389

Magnier, E. A., et al. 2013, ApJS, 205, 20

Maoz, D., Badenes, C., \& Bickerton, S. J. 2012, ApJ, 751, 143

Margala, D., Kirkby, D., Dawson, K., et al. 2016, ApJ, 831, 157

Massaro, F., Masetti, N., D’Abrusco, R., Paggi, A., \& Funk, S. 2014, AJ, 148, 66

McGraw, S. M., Brandt, W. N., Grier, C. J., et al. 2017, ArXiv e-prints, arXiv:1705.03019

Merloni, A., Predehl, P., Becker, W., et al. 2012, ArXiv e-prints, arXiv: 1209.3114

Metcalfe, N., Farrow, D. J., Cole, S., et al. 2013, MNRAS, 435, 1825

Morgan, D. P., West, A. A., Garcés, A., et al. 2012, AJ, 144, 93

Morganson, E., Green, P. J., Anderson, S. F., et al. 2015, ApJ, 806,244

Murray, N., Chiang, J., Grossman, S. A., \& Voit, G. M. 1995, ApJ, 451, 498

Myers, A. D., Palanque-Delabrouille, N., Prakash, A., et al. 2015, ApJS, 221, 27

Nebot Gómez-Morán, A., Gänsicke, B. T., Schreiber, M. R., et al. 2011, A\&A, 536, A43

Osterbrock, D. E., \& Mathews, W. G. 1986, ARA\&A, 24, 171

Palanque-Delabrouille, N., Magneville, C., Yèche, C., et al. 2016, A\&A, 587, A41

Palaversa, L., Ivezić, Ž., Eyer, L., et al. 2013, AJ, 146, 101

Pâris, I., Petitjean, P., Ross, N. P., et al. 2017, A\&A, 597, A79

Peterson, B. M. 1993, PASP, 105, 247

Popović, L. Č., Shapovalova, A. I., Ilić, D., et al. 2011, A\&A, 528, A130

Popović, L. v., Shapovalova, A. I., Ilić, D., et al. 2014, A\&A, 572, A66

Prakash, A., Licquia, T. C., Newman, J. A., et al. 2016, ApJS, 224, 34

Proga, D. 2000, ApJ, 538, 684

Rafiee, A., Pirkola, P., Hall, P. B., et al. 2016, MNRAS, 459, 2472 
Raichoor, A., Comparat, J., Delubac, T., et al. 2017, ArXiv e-prints, arXiv: 1704.00338

Reiners, A., \& Basri, G. 2008, ApJ, 684, 1390

Richards, G. T., Fan, X., Newberg, H. J., et al. 2002, AJ, 123, 2945

Richards, G. T., Kruczek, N. E., Gallagher, S. C., et al. 2011, AJ, 141, 167

Roig, B., Blanton, M. R., \& Ross, N. P. 2014, ApJ, 781, 72

Ruan, J. J., Anderson, S. F., Green, P. J., et al. 2016a, ApJ, 825, 137

Ruan, J. J., Anderson, S. F., Cales, S. L., et al. 2016b, ApJ, 826, 188

Runnoe, J. C., Cales, S., Ruan, J. J., et al. 2016, MNRAS, 455, 1691

Runnoe, J. C., Eracleous, M., Pennell, A., et al. 2017, MNRAS (in press)

Schlafly, E. F., Finkbeiner, D. P., Jurić, M., et al. 2012, ApJ, 756,158

Schmidt, S. J., Hawley, S. L., West, A. A., et al. 2015, AJ, 149, 158

Schmidt, S. J., West, A. A., Hawley, S. L., \& Pineda, J. S. 2010, AJ, 139, 1808

Schneider, D. P., Hall, P. B., Richards, G. T., et al. 2007, AJ, 134,102

Schneider, D. P., Richards, G. T., Hall, P. B., et al. 2010, AJ, 139, 2360

SDSS Collaboration, Albareti, F. D., Allende Prieto, C., et al. 2016, ArXiv e-prints, arXiv:1608.02013

Sergeev, S. G., Nazarov, S. V., \& Borman, G. A. 2017, MNRAS, 465, 1898

Sergeev, S. G., Pronik, V. I., \& Sergeeva, E. A. 2000, A\&A, 356, 41

Shen, Y., Liu, X., Loeb, A., \& Tremaine, S. 2013, ApJ, 775, 49

Shen, Y., Richards, G. T., Strauss, M. A., et al. 2011, ApJS, 194, 45

Shen, Y., Brandt, W. N., Dawson, K. S., et al. 2015, ApJS, 216, 4

Si, J., Luo, A., Li, Y., et al. 2014, Science China Physics, Mechanics, and Astronomy, 57, 176

Smee, S. A., Gunn, J. E., Uomoto, A., et al. 2013, AJ, 146, 32
Sowinski, L. G., Schmidt, G. D., \& Hines, D. C. 1997, in Astronomical Society of the Pacific Conference Series, Vol. 128, Mass Ejection from Active Galactic Nuclei, ed. N. Arav, I. Shlosman, \& R. J. Weymann, 305

Srianand, R., Hussain, T., Noterdaeme, P., et al. 2016, MNRAS, 460, 634

Storchi-Bergmann, T., Nemmen da Silva, R., Eracleous, M., et al. 2003, ApJ, 598, 956

Strateva, I. V., Strauss, M. A., Hao, L., et al. 2003, AJ, 126, 1720

Stubbs, C. W., et al. 2010, ApJS, 191, 376

Sun, M., Trump, J. R., Shen, Y., et al. 2015, ApJ, 811, 42

Tonry, J. L., et al. 2012, ApJ, 750, 99

Trump, J. R., Hall, P. B., Reichard, T. A., et al. 2006, ApJS, 165,1

Tsalmantza, P., Decarli, R., Dotti, M., \& Hogg, D. W. 2011, ApJ, 738, 20

Turnshek, D. A. 1988, in QSO Absorption Lines: Probing the Universe, ed. J. C. Blades, D. A. Turnshek, \& C. A. Norman, 17

VanderPlas, J. T., \& Ivezić, Ž. 2015, ApJ, 812, 18

Vivek, M., Srianand, R., Petitjean, P., et al. 2014, MNRAS, 440, 799

Wang, L., Greene, J. E., Ju, W., et al. 2017, ApJ, 834, 129

Waters, T., Kashi, A., Proga, D., et al. 2016, ApJ, 827, 53

West, A. A., Hawley, S. L., Bochanski, J. J., et al. 2008, AJ, 135, 785

West, A. A., Morgan, D. P., Bochanski, J. J., et al. 2011, AJ, 141,97

Weymann, R. J., Morris, S. L., Foltz, C. B., \& Hewett, P. C. 1991, ApJ, 373, 23

White, R. L., Becker, R. H., Gregg, M. D., et al. 2000, ApJS, 126,133

York, D. G., Adelman, J., Anderson, Jr., J. E., et al. 2000, AJ, 120,1579

Zhang, N.-X., Brandt, W. N., Ahmed, N. S., et al. 2017, ApJ, 839, 101 